\title{
4 Problematische Wirkungen der Mediennutzung
}

Die Diskussion über interindividuell stabile Wirkungen der Mediennutzung ist seit jeher fester Bestandteil der gesellschaftlichen und wissenschaftlichen Diskurse über die Medien. Gleichs These, die intensive Beschäftigung mit Effekten der Mediennutzung resultiere aus einer "Urfurcht vor den Wirkungen der Medien" in der Bevölkerung [Kursivsetzung im Original] (Gleich, 2004, S. 588), die Furcht vor schädlichen Medienwirkungen erhalte durch die Entwicklung neuer Medien immer wieder neue Nahrung, wobei die vorgebrachten Argumente immer die gleichen seien (ebenda), ist jedoch in dieser sehr verallgemeinernden Form nicht zuzustimmen. So kann Gleich entgegengesetzt werden, dass zwar mit Einführung neuer Medien neue Medienwirkungsdebatten $\mathrm{zu}$ beobachten sind, dass jedoch die These von der Gleichheit der Argumente kaum zutreffen kann, etablierten sich neue Medien doch in historisch jeweils einzigartigen gesellschaftlichen Konstellationen mit jeweils einzigartigen sozialen, gesetzlichen und wirtschaftlichen Bedingungen (vgl. auch Kleimann, 2007). Das Deutschland des späten 20. und frühen 21. Jahrhunderts, in dem etwa über Wirkungen, Gefahren und Nutzen des Internets oder der Computerspiele diskutiert wird, ist eine andere Gesellschaft als diejenige, in der der erste literarische Selbstmord, der erste Filmkuss oder der erste „Zombiestreifen“ diskutiert wurde. Obwohl die Debatten der Vergangenheit ähnliche Grundmuster aufwiesen, indem beispielsweise immer wieder über Gewaltdarstellungen oder sittliche Tabubrüche diskutiert wurde, waren sie immer auch differenziert und auf die Besonderheiten des neuen Mediums ausgerichtet.

Die folgende Darstellung der aktuellen Medienwirkungsdebatte dient dem Zweck, Notwendigkeiten gesellschaftlichen und insbesondere pädagogischen Handelns bezüglich kindlicher und jugendlicher Mediennutzung auszuleuchten. Insofern sollen nachfolgend nur solche Medienwirkungsdimensionen dargestellt werden, die einen klaren Bezug zur Erfahrungswelt und zur Entwicklung Minderjähriger haben. Dabei wird nach der Systematik vorgegangen, nicht nach einzelnen Medien oder bestimmten medialen Inhalten $\mathrm{zu}$ differenzieren, sondern zwischen den Resultaten medialer Wirkprozesse, den "abhängigen Variablen" zu trennen. Ein zentrales Problem bei der Erforschung von Medienwirkungen und insbesondere von negativen Medienwirkungen stellt die Tatsache dar, dass Mediennutzung und insbesondere problematische Mediennutzung als recht zuverlässiger Indikator für das Vorhandensein einer ganzen Reihe von Risiko- 
faktoren etwa auf die Leistungsentwicklung, die Sozialisation, oder die Gesundheit von Kindern und Jugendlichen gelten kann, ohne dass die Art der Mediennutzung zwingend kausal mit diesen Risikofaktoren verknüpft sein muss. Es werden aus diesem Grund nachfolgend insbesondere solche Studien dargestellt, die versuchen, durch ihr Forschungsdesign (Längsschnittstudien oder Experimentalstudien oder ihre Auswertungsmethodik (beispielsweise Partialkorrelationen oder komplexe Strukturgleichungsmodelle) eigenständige Medieneffekte herauszuarbeiten und Drittvariableneinflüsse weitgehend zu isolieren.

\subsection{Mediennutzung und schulische Leistung bzw. Leistungsfähigkeit}

Die Zusammenhänge zwischen der Nutzung elektronischer Medien und der schulischen Leistung von Kindern und Jugendlichen sind in seit den 50er-Jahren des letzten Jahrhunderts vielfach quer- und längsschnittlich untersucht worden. Dabei bezog sich die frühe Forschung ausschließlich auf Effekte des sich immer stärker verbreitenden Fernsehens, während Studien seit Mitte der 1980er-Jahre auch Effekte der Nutzung anderer elektronischer Medien (Video, Computerspiele, später auch Internet) thematisierten (Für eine ausführliche Darstellung der bisherigen Forschung vgl. Mößle et al., 2007).

\subsubsection{Schulleistung - Begriffsbestimmung und Beschreibung wichtiger Einfluss- faktoren}

Bevor ein kurzer Überblick über den aktuellen Forschungsstand gegeben wird, soll zunächst kurz der Begriff der schulischen Leistung erläutert werden. Schulleistungen werden von Helmke und Weinert definiert als erworbene bereichsspezifische Kenntnisse, Fertigkeiten und Leistungen, die durch schulbezogene Aufgaben und Leistungstests überprüfbar sind (1997, S. 139). Als zentrale Indikatoren schulbezogener Leistungen gelten dabei Leistungen, die sich aus Tests oder Lehrerbeurteilungen ergeben und in Form von Schulnoten vorliegen (ebenda). Neuere kompetenzorientierte Ansätze der Schulleistungsforschung rekurrieren dabei stärker auf den Erwerb langfristiger Expertise, die sich nicht nur auf den Erwerb bereichsspezifischer Wissensbestände bezieht, sondern auch die Kompetenz zur Anwendung dieser Wissensbestände umfasst. Damit lösen sie sich von der engen Auffassung von Schulleistung als durch den Lehrplan bedingte Verfügbarkeit bereichsspezifischen Wissens und betonen die Relevanz kognitiver Fähigkeiten und Fertigkeiten zur Lösung spezifischer Probleme 
(Köller \& Baumert, 2008; vgl. auch Rehbein, 2010). Insofern gibt es zumindest zwei unterschiedliche Ansätze, schulbezogene Leistungen zu messen, die in Studien verwendet werden können, welche den Einfluss verschiedener Faktoren (zum Beispiel der Mediennutzung) auf schulbezogene Leistungen untersuchen sollen: Die Messung solcher Leistungsindikatoren, die von der Schule selbst generiert werden (Schulnoten und Leistungszertifikate) und die Messung durch die Schule vermittelter (Basis-)Kompetenzen in Form allgemeiner Kompetenztests (zum Beispiel Lesekompetenzen, mathematische Grundbildung, naturwissenschaftliche Grundbildung, Fähigkeit zum selbstregulierten Lernen, soziale und kommunikative Kompetenzen)(Baumert, Stanat \& Demmrich, 2001). Baumert, Stanat und Demmrich verweisen allerdings - entgegen anderer Meinungen in der Literatur - darauf, dass Kompetenzen und schulische Bildungszertifikate durchaus miteinander zusammenhängen. Bildungszertifikate seien, so die Autoren, Kompetenzversprechen, die gegenüber Abweichungen im Einzelfall relativ enttäuschungsfest sind (Baumert, Stanat et al., 2001, S. 31). Zudem zeige die empirische Studienlage durchaus eine Kopplung zwischen formalen Bildungsindikatoren. So hingen Lesekompetenz und mathematische Fähigkeiten in ausgeprägter Weise mit dem erworbenen Bildungsniveau zusammen (ebenda).

Unstrittig ist, dass Schulleistung von einer Vielzahl von Faktoren bedingt und fortwährend beeinflusst ist. Helmke und Weinert unterscheiden hier individuelle, schulische und familiäre Bedingungsfaktoren sowie eine Gruppe „anderer Sozialisationsinstanzen“, zu der sie auch die Medien beziehungsweise Medienumwelt zählen (1997). Die Autoren stellen jedoch fest, dass die genauen $\mathrm{Zu}$ sammenhänge zwischen den einzelnen Faktoren und Schulleistung weitgehend unklar sind (Helmke, A. \& Weinert, 1997, S. 139). Die aktuellen kompetenzerwerbsorientierten internationalen Programme zum Vergleich schulischer Leistungen, PISA (Programme for International Student Assessment) und IGLU (Internationale Grundschul-Lese-Untersuchung), berücksichtigten in der Vergangenheit Medien und Mediennutzung von Schülern zunächst lediglich durch Erhebung der Ausstattung mit Mediengeräten (Baumert, Artelt, Carstensen, Sibberns \& Stanat, 2001) sowie Abfrage außerschulischer PC-Nutzung von Schülerinnen und Schülern (Bos et al., 2003), obwohl Medien und ihrer Nutzung im diesen Studien zugrundeliegenden Modell bereits Zusammenhänge mit kognitiven, motivationalen und sozialen Lernvoraussetzungen eines Schülers, seinem kulturellen Kapital, den Beziehungen mit seiner Peergroup sowie dem elterlichen Erziehungs- und Unterstützungsverhalten bescheinigt werden (Baumert, Artelt et al., 2001). Insofern „hinkt“ die empirische Operationalisierung dieser Studien dem zugrundeliegenden Kompetenzmodell 
deutlich hinterher, zumal die bisherigen mediennutzungsspezifischen Auswertungen insbesondere der PISA-Studie durchaus als kritikwürdig gelten können (vgl. S. 75 dieser Arbeit).

\subsubsection{Mediennutzung und Schulleistung: Die Forschung seit 1950}

Deutlich intensiver als die „klassische“ Schulleistungsforschung hat sich insbesondere die Medienwirkungsforschung und die Leseforschung der vergangenen Jahrzehnte mit dem Einfluss der Medien auf die Schulleistung beschäftigt. Hier dominierten zunächst Studien zur Wirkung der Fernsehnutzung, innerhalb derer der Fokus auf mögliche negative Effekte der Mediennutzung auf schulische Leistung und verwandte Konstrukte gelegt wurde (vgl. etwa Ennemoser, Schiffer, Reinsch \& Schneider, 2003, S. 12). Frühe Studien aus den 1950er- und 1960er-Jahren konnten indes entgegen der Annahme negativer Wirkungen keine, oder aufgrund der Effektstärke kaum relevante negative Effekte der Fernsehnutzung zeigen (ebenda), auch solche mit elaborierten Längsschnittdesigns nicht (vgl. etwa die Studie mit Daten aus den 60er-Jahren von Gortmaker, Salter, Walker \& Dietz, 1990). Auch die Meta-Analyse von Williams, Haertel, Haertel und Walberg (1982), in der 274 Korrelationen zwischen Freizeitmediennutzung und schulischem Lernen aus 23 Studien zwischen 1954 und 1978 systematisch analysiert und zusammengefasst wurden, zeigte einen lediglich äußerst schwachen, wenn auch signifikanten negativen $\mathrm{Zu}$ sammenhang von $r=.05$ zwischen Fernsehnutzung und einem schulischen Leistungsindikator. Als eine der zentralen Studien der Fernsehwirkungsforschung gilt die Studie „The Impact of Television“, in der Tannis MacBeth Williams und Kollegen den - für die Forscher - glücklichen Umstand nutzten, dass eine kanadische Kommune erst 1973 Fernsehempfang erhielt - nicht aufgrund einer besonderen geographischen Isolation, sondern aufgrund ihrer Lage in einem „Blinden Fleck“ der bisher vorhandenen Fernsehsendestationen - und die Forscher somit in der Lage waren, die Bewohner der Gemeinde, Kinder und Erwachsene vor und nach Einführung des Fernsehens zu untersuchen (vgl. Williams, T. M., 1986) ${ }^{34}$. Die von den Autoren der Studie gefundenen negativen

34 Die Einführung des Fernsehens in einer ansonsten durchschnittlichen kanadischen Kommune zu diesem späten Zeitpunkt nutzten die Forscher, die Bewohner dieser „Notel“ genannten Kommune, Kinder wie Erwachsene, vor und nach Einführung des Fernsehens intensiv zu Leseleistungen, kognitiver Entwicklung, kognitiver Leistungsfähigkeit, Freizeitgestaltung, sonstiger Mediennutzung, Geschlechtsrollenbildern, Gewaltverhalten sowie einiger weiterer Persönlichkeitsvariablen und Einstellungen zu untersuchen. Als 
Effekte des Fernsehens auf den Erwerb von Lesefähigkeiten, Leseverhalten und kreatives Denken stellten den Auftakt einer Reihe von Studienergebnissen der 1980er-Jahre dar, die Belege für negative Effekte der Fernsehnutzung auf schulische Leistungsparameter erbrachten ${ }^{35}$. Eine Meta-Analyse von Razel (2001), in die 305 Korrelationskoeffizienten zu Zusammenhängen zwischen Fernsehnutzung und Schulleistung aus sechs internationalen Studien von 1986 bis 1998 mit $N>1.000 .000$ Schülerinnen und Schülern einbezogen wurden, zeigte, dass 90 Prozent der dokumentierten Korrelationen zwischen Fernsehzeit und Schulleistung (Lesen, Mathematik und Naturwissenschaften) negativ ausfielen $^{36}$. Das kritische Review der Fernsehwirkungsforschung von Comstock und Scharrer (1999) kommt unter Rückgriff auf ein Zitat von Marie Winn (1977) zu dem Schluss, dass insbesondere eine negative Wirkung von Fernsehnutzung auf Lesefähigkeiten von Kindern nachgewiesen seien: „[...] large amounts of television viewing interfered with learning to read, the ability to concentrate while reading, and promoted the use of reading matter that could be scanned or read in brief segments with low involvement."

\subsubsection{Wirkungstheorien}

Welche theoretischen Annahmen existieren - jenseits der statistischen Assoziation zweier Sachverhalte - über die Art des Zusammenhangs zwischen Fernsehnutzung und schulischer Leistung? In der Forschung der vergangenen Jahrzehnte konkurrieren mehrere Hypothesen in der Debatte über negative Effekte des Fernsehens auf die schulische Leistung. In einer ersten Gruppe

Kontrollgruppen dienten zwei weitere, mit „Notel“" weitgehend vergleichbare kanadische Gemeinden, in denen, wie später auch in „Notel“, bereits ein Fernsehsender verfügbar war (,Unitel“"), oder bereits vier Fernsehsender (,Multitel“") (Williams, T. M., 1986, S. 1 8).

35 Die Resultate der kanadischen Studie bezüglich des Erwerbs der Lesefähigkeit von Kindern wurden unter anderem von Anderson et al. (2001) zunächst recht überzeugend relativiert. So verweisen die Autoren zu Recht darauf, dass sich der Fernseheffekt der Studie von Williams et al. vor allem durch die Neueinführung des Fernsehens erklären ließe, der nach Auftreten einer „,novelty bubble“ wieder verschwinde. Insofern werden die entsprechenden Ergebnisse von Williams et al. an dieser Stelle nicht weiter ausgeführt, insbesondere weil inzwischen überzeugendere Belege einer Beeinträchtigung von Leseerwerb und Leseverhalten von Kindern durch aktuelle Längsschnittstudien vorliegen, für die Anderson et al.'s Argumentation einer „,novelty bubble“ nicht mehr gelten kann.

$36 \mathrm{Zu}$ den Einschränkungen und Differenzierungen dieses Befundes vgl. Razel, 2001, S. $373-378$. 
können all jene Hypothesen und Theorien zusammengefasst werden, die sich mit aus Mediennutzung resultierenden Verdrängungseffekten beschäftigen. Zeitverdrängungshypothesen stellen auf die zunehmende mit (elektronischen) Medien verbrachte Zeit ab und folgern daraus die Verdrängung anderer, unter anderem schulnotwendiger Tätigkeiten, insbesondere die Verdrängung schulischen Lernens, der Erledigung von Hausaufgaben oder auch ein vermindertes Engagement für andere leistungsförderliche Freizeittätigkeiten (entsprechende Überblicke bei Comstock, G. A. \& Scharrer, 1999; Ennemoser \& Schneider, 2007; Mößle et al., 2007, S. 22 - 31; Neuman, 1988). E

Eine weitere Gruppe von Hypothesen fokussiert die von Kindern und Jugendlichen genutzten Medieninhalte und stellt einen Zusammenhang her zwischen der Kumulierung eher kurz- oder mittelfristiger Medienrezeptionseffekte und der schulischen Leistung. Elaborierte Ausarbeitungen dieser Medieninhaltshypothesen liegen insbesondere zu Effekten der Gewaltmediennutzung auf das Sozial- und Konfliktverhalten von Kindern und Jugendlichen vor und den Implikationen, die sich hieraus für schulische Leistungen ergeben (vgl. Mößle et al., 2007, S. 41 - 43).

Comstock und Scharrer zeigen, dass sich viele medienbezogene Schulleistungsminderungs-hypothesen in einer dritten, eher heterogenen Gruppe von Interferenz-Hypothesen zusammenfassen lassen (Comstock, G. A. \& Scharrer, 1999, S. 255 - 256), wobei in dieser Kategorie sowohl Zeitverdrängungs- wie auch Medieninhaltsanteile enthalten sind. Die Gruppe der Interferenz-Hypothesen schließt auch solche Überlegungen ein, die sich auf Minderung bestimmter kognitiver Leistungsdimensionen von Rezipienten beziehen, die sich durch Bevorzugung bestimmter Präsentationsformate durch das Fernsehen erklärt werden sollen. Nach Comstock und Scharrer finden sich in der Fernsehnutzungsforschung der vergangenen Jahrzehnte empirische Belege dafür, dass schulbezogene Tätigkeiten durch Fernsehnutzung nicht oder nur sehr moderat verdrängt werden, dass aber parallele Fernsehnutzung bei der Erledigung schulbezogener Aufgaben (zum Beispiel Fernsehnutzung beim Erledigen von Hausaufgaben oder während des Lernens) nachweisbar negative Effekte hat (vgl. auch Armstrong \& Greenberg, 1990). Auch finden Comstock und Scharrer in der Fernsehforschung der Vergangenheit Belege dafür, dass kindliche Vielseher früh fernsehtypische Narrationsweisen, Plots und Präsentationsweisen präferieren und typische Narrationsweisen, Plots und Präsentationen gedruckter Geschichten im Laufe der Zeit immer stärker als farblos, langweilig und wertlos ablehnen. In einer immer noch stark am Lesen und textbasierter Inhaltsvermittlung orientierten Schule erzielen demzufolge solche Schülerinnen und Schüler bessere Leistungserfolge, die im Umgang mit ge- 
druckten Inhalten geübter sind und diese Art der Vermittlung stärker wertschätzen (Comstock, G. A. \& Scharrer, 1999, S. 261 - 264; vgl. auch Koolstra \& Van der Voort, 1996). Andere auf den Medieninhalt bezogene Theorien, wie etwa die der direkten Konzentrationsfähigkeitsminderung durch starke Fernsehnutzung (Gadberry, 1980) konnten bisher nicht empirisch bestätigt werden (Ennemoser \& Schneider, 2007, S. 366). Vielmehr erscheint es plausibel, dass die Konzentrationsfähigkeit durch starke frühe Fernsehnutzung nicht dauerhaft gemindert wird, sondern bei Vielsehern eine geringe Motivation besteht, ihre Aufmerksamkeit auf von ihnen als eher minderwertig angesehene, gedruckte Medieninhalte zu lenken.

Insgesamt muss jedoch darauf verwiesen werden, dass die Trennung zwischen Zeitverdrängungshypothesen, medieninhaltsbezogenen Hypothesen und Interferenzhypothesen eher das Resultat nachträglicher Versuche darstellt, die bisherige Forschung zu strukturieren, als dass sich die Hypothesen bei den relevanten Autoren wirklich trennscharf unterscheiden lassen. Da die Schulleistungsminderung durch Mediennutzung nur als langfristiger Effekt denkbar ist und nach Hypothesen differenzierte Experimentalstudien kaum denkbar erscheinen, lassen sich die konkurrierenden Hypothesen kaum empirisch gegeneinander testen. So können empirische Studien lediglich versuchen, die Kausalität des Effektes von Medien (insbesondere Fernsehnutzung) auf Schulleistung nachzuweisen, nicht aber die genauen Effekte erklären.

\subsubsection{Fernsehnutzung und Schulleistung}

Die eindeutigste empirische Studienlage ergibt sich auf dem Feld der Forschung zum Zusammenhang zwischen Fernsehnutzung und Lesefähigkeiten von Kindern. Die publizierten Studien stellen selten keine, zumeist aber negative Effekte der Fernsehnutzung in der Vor- und Grundschulzeit auf die Leseleistungen beziehungsweise -fähigkeiten fest. Konnten ältere Studien nur geringe (vgl. die Meta-Analyse von Williams, P. A. et al., 1982) oder, wie die Längsschnittstudie von Gortmaker, Salter, Walker und Dietz (1990), die auf Daten aus den 1960er-Jahren zurückgreift, keine Effekte feststellen, liegen inzwischen drei neuere elaborierte Studien im Längsschnittdesign ${ }^{37}$ aus den USA, aus Deutsch-

37 Obwohl forschungsorganisatorisch und forschungsökonomisch extrem aufwändig, haben Längsschnittanalyse doch den großen Vorteil gegenüber reinen Querschnittsuntersuchungen, dass - mit adäquatem Analyseinstrumentarium - statistische Zusammenhänge zwischen Variablen mehrerer Messzeitpunkte eindeutig kausal interpretiert werden 
land und den Niederlanden mit recht konsistenten Ergebnissen vor, die durchaus signifikant negative Effekte des Fernsehens auf Lesefähigkeiten von Kindern feststellen.

Tabelle 1: Längsschnittstudien zum Einfluss des Fernsehen auf Lesefähigkeiten von Kindern

\begin{tabular}{|c|c|c|c|}
\hline & $\begin{array}{l}\text { Koolstra, van der } \\
\text { Voort \& van der } \\
\text { Kamp (1997) }\end{array}$ & $\begin{array}{l}\text { Zimmerman \& } \\
\text { Christakis (2005) }\end{array}$ & $\begin{array}{l}\text { Ennemoser } \\
(2003 a), \\
\text { Ennemoser \& } \\
\text { Schneider (2007) }\end{array}$ \\
\hline Forschungsdesign & Längsschnitt & Längsschnitt & Längsschnitt \\
\hline $\begin{array}{l}\text { Untersuchungszeit- } \\
\text { raum }\end{array}$ & $1989-1991$ & $1994-2000$ & $1998-1999$ \\
\hline $\begin{array}{l}\text { Untersuchungs- } \\
\text { region }\end{array}$ & Niederlande & USA & Süddeutschland \\
\hline $\begin{array}{l}\text { Alter der Unter- } \\
\text { suchungsteilnehmer }\end{array}$ & 8 - 10 Jahre & 0-7 Jahre & 5 - 10 Jahre \\
\hline Stichprobengröße & $\mathrm{N}=1.050$ & $\mathrm{~N}=1.797$ & $\mathrm{~N}=332$ \\
\hline Stichprobenziehung & $\begin{array}{l}\text { Quotierte Stichprobe } \\
\text { von Kindern in } 16 \\
\text { Schulen Südhollands }\end{array}$ & $\begin{array}{l}\text { Randomisiert } \\
\text { (Oversampling für } \\
\text { bestimmte } \\
\text { ethnische Gruppen) }\end{array}$ & $\begin{array}{l}\text { Anfallende Stich- } \\
\text { probe }\end{array}$ \\
\hline Erhebungsmethode & $\begin{array}{l}\text { Standardisierte, } \\
\text { schriftliche Be- } \\
\text { fragung im Klassen- } \\
\text { verband }\end{array}$ & $\begin{array}{l}\text { Postalisch- } \\
\text { schriftliche } \\
\text { standardisierte } \\
\text { Interviews }\end{array}$ & $\begin{array}{l}\text { Persönliche } \\
\text { standardisierte } \\
\text { Interviews, } \\
\text { standardisierte } \\
\text { Tests, } \\
\text { Selbstausfüller- } \\
\text { Medientagebuch }\end{array}$ \\
\hline
\end{tabular}

können, ohne dabei den Beschränkungen der ebenfalls zur Kausalanalyse geeigneten experimentellen Verfahren zu unterliegen (vgl. Pfeiffer, C., Mößle, Kleimann \& Rehbein, 2008). 
Tablle 1 (fortgesetzt)

\begin{tabular}{|c|c|c|c|}
\hline & $\begin{array}{l}\text { Koolstra, van der } \\
\text { Voort \& van der } \\
\text { Kamp (1997) }\end{array}$ & $\begin{array}{l}\text { Zimmerman \& } \\
\text { Christakis (2005) }\end{array}$ & $\begin{array}{l}\text { Ennemoser } \\
(2003 a), \\
\text { Ennemoser \& } \\
\text { Schneider (2007) }\end{array}$ \\
\hline $\begin{array}{l}\text { Operationalisierung } \\
\text { der Fernsehnutzung }\end{array}$ & $\begin{array}{l}\text { Fernsehnutzungs- } \\
\text { dauer und } \\
\text {-inhalt (Unterhaltung } \\
\text { und Information) } \\
\text { (Selbstauskunft) }\end{array}$ & $\begin{array}{l}\text { Fernsehnutzungs- } \\
\text { dauer (Fremdaus- } \\
\text { kunft Mutter) }\end{array}$ & $\begin{array}{l}\text { Fernsehnutzungs- } \\
\text { dauer (Fremdaus- } \\
\text { kunft durch Eltern } \\
\text { mittels Medien- } \\
\text { tagebuch; Selbst- } \\
\text { auskunft mittels } \\
\text { Medientagebuch) }\end{array}$ \\
\hline $\begin{array}{l}\text { Gemessene } \\
\text { Dimensionen } \\
\text { schulischer } \\
\text { Leistungsfähigkeit }\end{array}$ & $\begin{array}{l}\text { Leseverständnis } \\
\text { Text-Dekodierungs- } \\
\text { fähigkeiten }\end{array}$ & $\begin{array}{l}\text { Fähigkeiten in der } \\
\text { Texterkennung } \\
\text { Fähigkeiten im } \\
\text { Leseverständnis } \\
\text { Zusätzlich: } \\
\text { Mathematische } \\
\text { Fähigkeiten }\end{array}$ & $\begin{array}{l}\text { Lesegeschwindig- } \\
\text { keit } \\
\text { Leseverständnis } \\
\text { Wortschatz } \\
\text { Allgemeiner } \\
\text { sprachlicher Ent- } \\
\text { wicklungsstand }\end{array}$ \\
\hline $\begin{array}{l}\text { Kontrollierte } \\
\text { Variablen }\end{array}$ & $\begin{array}{l}\text { Intelligenz der } \\
\text { Kinder } \\
\text { Sozioökonomischer } \\
\text { Status der Eltern } \\
\text { (inkl. Bildungs- } \\
\text { hintergrund) }\end{array}$ & $\begin{array}{l}\text { Ethnischer Hinter- } \\
\text { grund der Kinder } \\
\text { Kognitive } \\
\text { Stimulation durch } \\
\text { Eltern } \\
\text { Sprachfähigkeiten } \\
\text { der Eltern } \\
\text { Bildungshinter- } \\
\text { grund der Mutter } \\
\text { Intelligenz der } \\
\text { Mutter }\end{array}$ & $\begin{array}{l}\text { Intelligenz der } \\
\text { Kinder } \\
\text { Schulabschluss der } \\
\text { Eltern } \\
\text { Berufsausbildung } \\
\text { der Eltern } \\
\text { Derzeitige Tätig- } \\
\text { keit der Eltern }\end{array}$ \\
\hline
\end{tabular}


Tablle 1 (fortgesetzt)

\begin{tabular}{|c|c|c|c|}
\hline & $\begin{array}{l}\text { Koolstra, van der } \\
\text { Voort \& van der } \\
\text { Kamp (1997) }\end{array}$ & $\begin{array}{l}\text { Zimmerman \& } \\
\text { Christakis (2005) }\end{array}$ & $\begin{array}{l}\text { Ennemoser } \\
(2003 a), \\
\text { Ennemoser \& } \\
\text { Schneider (2007) }\end{array}$ \\
\hline Hauptresultate & $\begin{array}{l}\text { Negativer Einfluss } \\
\text { der Häufigkeit von } \\
\text { TV- } \\
\text { Unterhaltungs- } \\
\text { konsum auf das } \\
\text { Leseverständnis. } \\
\text { Positiver Einfluss der } \\
\text { häufigen Rezeption } \\
\text { ausländischer Filme } \\
\text { mit Untertiteln auf } \\
\text { das Leseverständnis }\end{array}$ & $\begin{array}{l}\text { Negativer Einfluss } \\
\text { frühen Fernseh- } \\
\text { konsums (im Alter } \\
\text { von unter drei } \\
\text { Jahren) auf } \\
\text { schulnahe } \\
\text { kognitive } \\
\text { Leistungen }\end{array}$ & $\begin{array}{l}\text { Negativer Einfluss } \\
\text { häufigen Fernseh- } \\
\text { konsums auf } \\
\text { Sprach- und Lese- } \\
\text { kompetenzen }\end{array}$ \\
\hline
\end{tabular}

Koolstra, van der Voort und van der Kamp (1997) (vgl. Tabelle 1) zeigten, dass die Häufigkeit der Fernsehnutzung bei Kontrolle der Intelligenz der Kinder sowie des elterlichen Hintergrunds zu schlechteren Testleistungen der Kinder beim Leseverständnis und der Dekodierung von Texten führte. Dieser Effekt zeigte sich vor allem für Kinder, die überwiegend Unterhaltungssendungen nutzten. Nur Kinder, die bereits im frühen Grundschulalter untertitelte Fernsehsendungen anschauten, profitierten davon im Hinblick auf bessere Lesefähigkeiten. Auch Zimmerman und Christakis (2005) kamen in Ihrer Sekundäranalyse des amerikanischen National Longitudinal Survey of Youth 1979 - Children and Young Adults (NLSY '79 und NLSY-Child) zu dem Schluss, dass ein linearnegativer Zusammenhang zwischen der Häufigkeit früher Fernsehnutzung im Alter von unter drei Jahren und den Lesefähigkeiten (Texterkennung und Leseverständnis) von Kindern im Alter von sechs bis sieben Jahren existiert. Für die mathematischen Fähigkeiten der Kinder, die in dieser Studie zusätzlich untersucht wurden, zeigten sich analoge Zusammenhänge. In Deutschland fanden Ennemoser, Schiffer, Reinsch und Schneider (Ennemoser, 2003b; Ennemoser et al., 2003; Ennemoser \& Schneider, 2007; Schiffer, Ennemoser \& Schneider, 2002) ebenfalls einen quer- wie längsschnittlich signifikanten negativen $\mathrm{Zu}$ sammenhang zwischen häufiger Unterhaltungsfernsehnutzung und schwächeren Sprach- und Lesekompetenzen. Die Autoren wiesen dabei insbesondere klare 
Effekte der Unterhaltungsfernsehnutzung in der ersten Klasse auf Lesekompetenzen in der dritten Klasse nach (Ennemoser \& Schneider, 2007, S. 363).

Gestützt werden die oben genannten Längsschnittergebnisse durch aktuelle Studien wie die von Borzekowski und Robinson (2005), die einen rein korrelativen Zusammenhang zwischen Leseleitung und Fernsehgerätebesitz von Drittklässlern fand, sowie durch weitere Studien, in deren Zentrum nicht allein die Lesefähigkeit von Kindern stand, sondern die allgemeinen schulischakademischen Leistungen von Kindern und Jugendlichen.

So legten Winterstein und Jungwirth (2006) Daten aus deutschen Einschulungsuntersuchungen von $\mathrm{N}=1894$ Vorschulkindern zwischen fünf und sechs Jahren vor, in denen sie Fernsehzeiten der Kinder und ihre Leistungen im Mensch-Zeichentest (MZT) korrelierten. Dabei zeigten vielsehende Kinder signifikant schlechtere Ergebnisse im MZT, der als guter Indikator der allgemeinen kognitiven Entwicklung von Kindern gilt (ebenda, S. 205). Neben solchen korrelativen Befunden ist auch auf eine Reihe von Längsschnittbefunden $\mathrm{zu}$ verweisen: Anderson, Huston, Schmitt, Linebarger und Wright (2001) publizierten eine Studie, in der sie von 1981 bis 1983 in zwei Kohorten von insgesamt $\mathrm{N}=655$ fünfjährigen Vorschulkindern die Fernsehnutzung die Kinder mithilfe von Fernsehtagebüchern erhoben ${ }^{38}$ und diese Daten 1994, also rund zwölf Jahre später, mit den Schulnoten der inzwischen durchschnittlich 17jährigen Schülerinnen und Schüler korrelierten. Die Autoren stellen insgesamt eindeutige Effekte vorschulischer Fernsehnutzung fest, sehen jedoch eine dominante Rolle des jeweiligen Inhalts der genutzten Fernsehsendungen, der von leicht positiven Effekten auf die Schulleistung bei primärer Nutzung informativer Programme bis $\mathrm{zu}$ deutlich negativen Effekten bei primärer Nutzung unterhaltsamer, insbesondere gewalthaltiger Fernsehsendungen reicht (S. 20 - 21). Eindeutig negative Effekte des Fernsehens auf schulische Leistungen konstatieren Hancox, Milne und Poulton (2005) die eine zwischen 1972 und 1973 geborene Geburtskohorte bis ins Alter von durchschnittlich 26 Jahren begleiteten, in diesem Zeitraum zu sechs Messzeitpunkten Fernsehnutzungsdaten sammelten und darüber hinaus differenzierte Daten zur schulischen Leistungsentwicklung, Schulabbruchquoten und akademischen Abschlüssen erhoben. Insgesamt, stellen sie fest, sei Fernsehnutzung in Kindheit und Jugend mit signifikant höheren Schulabbruchraten und schlechteren akademischen Abschlüssen im Alter von 26 Jahren assoziiert

38 Zur Eignung von Fernseh- bzw. Medientagebüchern zur validen Erfassung der Mediennutzung vgl. insbesondere Reinsch, Ennemoser und Schneider (1999), ebenso Anderson, Field, Collins, Lorch und Nathan (1985) sowie Bechtel, Achelpohl und Akers (1972). 
(S. 614). Dabei waren die Autoren in der Lage, den Intelligenzquotienten, den sozioökonomischen Status des Elternhauses und die Einschätzung von Lehrern und Eltern über verschiedene Verhaltensprobleme der Kinder zu kontrollieren.

Dass nicht nur die Lesefähigkeiten negativen Fernsehnutzungseffekten unterliegen, sondern auch schulische Leistungen in anderen Fächern, legen auch Erkenntnisse der oben bereits erwähnten Studien von Zimmerman und Christakis (2005) sowie von Borzekowski und Robinson (2005) nahe, da hier neben Leseleistungen jeweils auch mathematische Fähigkeiten getestet wurden. Für den deutschsprachigen Raum zeigte dies auch die KFN-Grundschulbefragung 2005, die - bei Kontrolle des elterlichen Bildungshintergrundes und des Familienklimas, der ethnischen Herkunft und der Deutschkenntnisse der Kinder bei Einschulung - negative Korrelationen für die drei Kernfächer Deutsch, Sachkunde und Mathematik mit der von den $\mathrm{N}=5.529$ befragten Viertklässlerinnen und Viertklässlern selbst eingeschätzten Fernsehzeit auswies (Mößle et al., 2007, S. 91 - 102). Der negative Effekt der Fernsehnutzung zeigte sich sowohl auf der zeitlichen (Nutzungsdauer) wie auch der inhaltlichen Ebene (Gewaltmedienanteil), und wurde sowohl bei den Schulnoten der Kinder als auch bei den Empfehlungen der Lehrerinnen und Lehrer für eine weiterführende Schule deutlich. Insgesamt weisen aktuelle Studien somit auf problematische Wirkungen der Fernsehnutzung im Vor- und Grundschulalter auf Leseleistungen wie auch auf allgemeine Schulleistung und die weitere akademische Laufbahn hin.

In der Literatur zu Medienwirkungen auf Schulleistungen wird immer wieder die Frage diskutiert, ob die gängige Praxis, Medieneffekte und insbesondere Effekte der Mediennutzungszeit auf Schulleistung als linearen Zusammenhang zu modellieren, überhaupt zulässig ist (Comstock, G. A. \& Scharrer, 1999; Ennemoser \& Schneider, 2007; Neuman, 1988; Razel, 2001; Williams, P. A. et al., 1982). So zeigte sich besonders in frühen Studien zur Wirkung der täglichen Fernsehnutzung die Tendenz eines kurvilinearen Zusammenhangs. Kinder und Jugendliche mit maßvoller Fernsehnutzung wiesen nicht nur bessere Schulleistungen auf als vielsehende Kinder, sondern auch leicht bessere Leistungen als Schülerinnen und Schüler, die den Fernseher gar nicht nutzten (Razel, 2001; Williams, P. A. et al., 1982). Andere Studien, etwa die Würzburger Längsschnittstudie von Ennemoser und Schneider fanden keinen kurvilinearen $\mathrm{Zu}$ sammenhang, sondern linear-negative Effekte (Ennemoser \& Schneider, 2007). Die zunächst methodische Diskussion über die genaue Art des Zusammenhangs ist insofern von Relevanz für die in dieser Arbeit behandelte Fragestellung, als die Frage der „richtigen Dosis“ kindlicher Mediennutzung im pädagogischen Kontext häufig diskutiert wird und besonders Eltern in der täglichen Erziehungspraxis immer wieder beschäftigt. Allerdings lässt sich die Relevanz dieser Frage 
durchaus auch anzweifeln, solange die ,richtige Dosis“ der Mediennutzung auf die reinen Medienzeiten beschränkt bleiben. Comstock und Scharrer bezweifeln daher die Relevanz der Diskussion um die genaue Art des Zusammenhangs und verweisen dabei auf den engen Zusammenhang zwischen Fernsehnutzungszeiten und verschiedenen inhaltlichen Nutzungsmustern.

Thus, light viewing by young persons is associated with more instrumental and information-oriented use that is unlikely to interfere with and might enhance scholastic performance, whereas heavy viewing reflects not only greater use but greater emphasis on less scholastically useful content (Comstock \& Scharrer, 1999, S 254).

Da die wenigsten Studien Mediennutzungszeiten und genutzte Medieninhalte getrennt differenziert voneinander erfassen, besteht somit die Möglichkeit, dass kurvilineare Zusammenhänge zwischen Mediennutzung und Schulleistung durch verschiedene inhaltliche Nutzungsmuster erklärt werden. Insofern spielt die Frage nach inhaltlichen Mediennutzungsmustern eine ganz wesentliche Rolle bei der Postulierung eines Zusammenhangs zwischen Mediennutzung und Schulleistung.

Können viele der oben beschriebenen Korrelationen zwischen Fernsehnutzung als Belege für die Zeitverdrängungshypothese angeführt werden, zeigen insbesondere Anderson et al. (2001), dass empirische Befunde auch überzeugend mit inhaltsbezogenen Theorien zu begründen sind. Schulische Leistungen sind, so die Überlegung, durch schriftliche und mündliche Leistungsmessungen validierte und durch Schulnoten dokumentierte Vermutungen von Lehrern über das Leistungsniveau einer Schülerin oder eines Schülers. Da die Prozesse der Leistungserbringung durch den Schüler und der Leistungsbewertung durch die Lehrkraft soziale Prozesse sind, die somit auch immer vom Verhältnis beider Personen bestimmt und zudem durch bestimmte Erwartungen auf beiden Seiten geprägt sind, beeinflusst ein auffälliges Sozialverhalten eines Schülers auch den Prozess der Notenvergabe. So kann beispielsweise Aggressivität auf Seiten eines Schülers zu einer Spirale von Ablehnung durch die Lehrkraft, weniger Leistung, geringerer Arbeitsqualität, schlechteren Noten, verringerter Erfolgsmotivation führen (Mößle et al., 2007, S. 41), die schließlich in einer Ablehnung der durch die schulisch vermittelte Lern- und Lernerfolgskultur mündet (vgl. auch Anderson, D. R. et al., 2001; MacBeth, 1996; Williams, T. M., 1986).

Ein genauer Blick in die Befunde der Einzelstudien zur Überprüfung der Inhaltshypothese zeigt jedoch insofern ein differenziertes Bild, als dass einzelne Studien besonders im Hinblick auf die genutzten Inhalte $\mathrm{zu}$ keineswegs konsistenten Ergebnissen kommen. So zeigen Anderson, Huston, Schmitt, Linebarger und Wright (2001), dass die Nutzung bestimmter Informations- und Infotainment-Inhalte im Vorschulalter bei Kontrolle wichtiger Einflussfaktoren 
leicht positiv mit schulischer Leistung im Jugendalter korreliert ist. Diese Erkenntnis korrespondiert im Übrigen mit Befunden von Linebarger und Walker (2005), die schon bei Kleinkindern positive Effekte bestimmter Sendungen auf Wortschatz und sprachliches Ausdrucksvermögen feststellten, während andere, ebenfalls auf Kleinkinder zugeschnittene Formate (wie etwa die Teletubbies) negative Effekte auf das sprachliche Ausdrucksvermögen und/oder den Wortschatz hatten. Bezüglich der Nutzung des seit Jahrzehnten sehr populären Edutainment-Formates „Sesamstraße“ durch Kinder im Klein- und Vorschulalter gibt es sehr unterschiedliche Befunde. Sie reichen von negativen Effekten der Nutzung dieses Formates auf das sprachliche Vermögen bis hin zu kleinen, aber robust positiven Effekten der Sesamstraßen-Nutzung auf Sprachlernen, Schulfähigkeit und spätere bessere Schulnoten (vgl. auch die von Comstock, G. A. \& Scharrer, 1999, zusammengefassten Befunde zu den positiven Effekten der Sesamstraße, S. 235 - 236; sowie Kleimann \& Mößle, 2006, S. 5). Darüber hinaus zeigten Koolstra, van er Voort und van der Kamp, dass die regelmäßige Rezeption von untertitelten Filmen durch Grundschulkinder deren Leseleistungen signifikant positiv beeinflusst (Koolstra et al., 1997).

Nicht nur zwischen Nutzungszeiten und genutzten Inhalten muss differenziert werden, auch die von den Kindern in den Nutzungsprozess eingebrachten Prädispositionen und Fähigkeiten sind nicht $\mathrm{zu}$ vernachlässigen (vgl. etwa die differenzierte Darstellung bei Ennemoser, 2003b). Klaren globalen Effekten stehen also differenzierte Effekte auf Subgruppenebene gegenüber, die sowohl mit der zeitlichen Mediennutzung als auch mit den genutzten Medieninhalten in Verbindung gebracht werden können. Trotz der Wichtigkeit solcher differenzierter Betrachtungen darf aber im Hinblick auf notwendiges gesellschaftliches Handeln und gebotene pädagogische Maßnahmen, die im Einzelnen im Rahmen dieser Arbeit noch zu diskutieren sind, das problematische Wirkpotential häufiger und inhaltlich problematischer Fernsehnutzung auf schulische Leistungen keinesfalls unterschätzt werden. So vielversprechend etwa die Nutzung einzelner Fernsehsendungen auf bestimmte Entwicklungsaspekte sein kann, zeigen Mediennutzungsstudien doch sehr eindeutig, dass solche eindeutig leistungsförderlichen Mediennutzungsmuster keinesfalls die Regel, sondern die klare Ausnahme sind. Zudem zeigen Studien wie die von Anderson, Huston, Schmitt, Linebarger und Wright (2001) oder von Linebarger und Walker (2005) immer nur den relativen Nutzen eines bestimmten Medieninhaltes gegenüber einem anderen. Aus entwicklungspsychologischer Sicht entscheidend wäre aber der Vergleich der Nutzung eher entwicklungsförderlicher Fernsehinhalte mit anderen Medien und vor allem auch nichtmedialen Aktivitäten und deren Wirkung. 


\subsubsection{Computer(-spiel-)nutzung und Schulleistung}

Neben den Effekten der Fernsehnutzung auf die schulische Leistung ist in den letzten Jahren der Einfluss interaktiver Computer- und Konsolenspiele auf die Schulleistung von Kindern verstärkt untersucht worden. Obwohl die Frage nach leistungsmindernden Effekten der Computerspielnutzung bereits durch Studien in den achtziger Jahren aufgeworfen wurde (Harris \& Williams, 1985; Lin \& Lepper, 1987), dominierte doch anfangs die auch durch empirische Studien belegte Hoffnung auf leistungsförderliche Effekte der Computerspielnutzung. David Walsh und Douglas Gentile fassen die Gründe für diese Hoffnungen wie folgt zusammen: "Video games are natural teachers. Children find them highly motivating; by virtue of their interactive nature, children are actively engaged with them; they provide repeated practice; and they include rewards for skillful play" (Walsh \& Gentile, 2004). Walsh und Gentile verweisen darauf, dass es mithilfe speziell entwickelter Spiele bereits gelungen sei, Einstellungen, Fähigkeiten und Verhalten von Kindern zu ändern, etwa im Bereich des Gesundheitsverhaltens. In der Tat ist inzwischen belegt, dass Computerspielen helfen kann, das räumliche Vorstellungsvermögen von Erwachsenen und auch Kindern zu verbessern (De Lisi \& Wolford, 2002; Green, C. Shawn \& Bavelier, 2006) und die visuelle Aufmerksamkeit zu steigern (Green, C. S. \& Bavelier, 2003; Risenhuber, 2004). Auch über den erfolgreichen Einsatz von Bildungssoftware, darunter zahlreiche Programme mit Computerspielcharakter, gibt es inzwischen zahlreiche Belege (vgl. die Metaanalyse von Murphy, Penuel, Means, Korbak \& Whaley, 2002).

Die PISA-Studie der OECD (2001) schien die positiven Zusammenhänge zwischen Computer(-spiel-) nutzung und schulischen oder schulnahen Leistungsindikatoren zunächst zu bestätigen, indem eine positive Korrelation zwischen höheren Lese- und Mathematikkompetenzen jugendlicher Schülerinnen und Schüler und der Verfügbarkeit eines Computers festgestellt wurde. In einer ReAnalyse der deutschen PISA-Daten durch Fuchs und Wößmann (2004) zeigte sich jedoch, dass bei Kontrolle des familiären Hintergrundes (insbesondere der elterlichen Erziehung und des sozioökonomischen Status) die Verfügbarkeit eines Computers zu einem umgekehrten Effekt, namentlich einer verminderten Lese- und Mathematikkompetenz, führte. Der Computerbesitz, so die Interpretation der Autoren, war Anfang des Jahrhunderts vor allem Indikator eines hohen sozioökonomischen Status, der wiederum mit einem höheren Bildungsniveau im Elternhaus und folglich besseren Ausgangsbedingungen für die Schullaufbahn ihrer Kinder verknüpft war. Wurde der Computer von den Schülern tatsächlich genutzt, so Fuchs und Wößmann, so diente er hauptsächlich als Spiele- 
plattform, was, so die These der Autoren im Sinne der Zeitverdrängungshypothese, eher vom Lernen ablenke als mathematische oder sprachliche Kompetenzen $\mathrm{zu}$ steigern. In ähnlicher Weise kann auch die Analyse von Wittwer und Senkbeil (2008) interpretiert werden, innerhalb derer sich die Autoren mit der Aussage der OECD auseinandersetzen, die Verfügbarkeit und häufige Nutzung eines Computers steigere die mathematischen Kompetenzen der in der zweiten PISA-Studie untersuchten Schülerinnen und Schüler (OECD, 2006). Wittwer und Senkbeil zeigen, dass bei Kontrolle wichtiger Faktoren wie Geschlecht, Migrationshintergrund, kognitiven Fähigkeiten sowie Fernsehnutzungshäufigkeit und Nutzung inhaltlich problematischer Filmgenres ${ }^{39}$ der Nutzung des Computers kein positiver, sondern ein (wenn auch nicht signifikanter) tendenziell eher negativer Einfluss zukommt (Wittwer \& Senkbeil, 2008, S. 1565 - 1566). Positive Effekte der Computernutzung auf die bei PISA 2003 getesteten Mathematik- und Problemlösungskompetenzen konnten dagegen nur für eine Gruppe von Schülern tatsächlich nachgewiesen werden: So genannte smart-users (18 Prozent der PISA-Stichprobe), die ein breites Spektrum der Computernutzung aufweisen (breite Nutzung sowohl für schulbezogene Tätigkeiten, Unterhaltungszwecke sowie zur Kommunikation) und sich diese Nutzung selbst angeeignet haben (S. 1566 - 1567).

Vor dem Hintergrund der hauptsächlichen Verwendung des Computers als Spielplattform und angesichts der Tatsache, dass unter den genutzten Spielen eher solche mit problematischen Inhalten als solche mit eindeutig entwicklungsoder leistungsförderlichen Inhalten genutzt werden (vgl. S. 36 und S. 43 in dieser Arbeit), erscheint es wenig erstaunlich, dass auch andere Studien, die sich nicht mit den Potentialen und Effekten von Lernspielen beschäftigen, sondern mit den alltäglichen Nutzungsvorlieben von Schülerinnen und Schülern, einhellig zu eher leistungsmindernden Effekten der Computerspielnutzung kommen. Gentile Lynch, Linder und Walsh (2004) stellen in einer Studie an $\mathrm{N}=607$ amerikanischen Acht- und Neuntklässlern eine negative Korrelation zwischen der Computerspielzeit und den durchschnittlichen Schulnoten der befragten Schülerinnen und Schüler fest. Auch Roberts, Foehr und Rideout (2005) fanden in einer für die USA repräsentativen Querschnittsbefragung von 2.023 Schülerinnen und Schülern der dritten bis zwölften Klasse negative Korrelationen zwischen Computerspielnutzung und der schulischen Leistung.

39 Dabei hat die Nutzung von Filmgenres wie Horror- oder Pornofilm bei Kontrolle der wichtiger Einflussfaktoren einen signifikant negativen Einfluss auf die Mathematik- und Problemlösungskompetenzen der untersuchten Schülerinnen und Schüler (Wittwer \& Senkbeil, 2008, S. 1566) 
Die Studie von Foehr und Rideout macht an mehreren Punkten deutlich, wie vergleichsweise wenig entwickelt der Forschungsstand zum Zusammenhang zwischen Computerspielen und Schulleistung im Gegensatz zur Fernsehforschung ist und wie dringend notwendig weitere Forschungsarbeit $\mathrm{zu}$ den Effekten der aktuellen Bildschirmmediennutzung von Kindern auf schulische Leistung ist. Zwar kann es generell als Stärke der Studie von Roberts Foehr und Rideout angesehen werden, dass im Rahmen ihrer Studie neben dem Computerspielen noch weitere für den Alltag von Schülerinnen und Schülern relevante Medien berücksichtigt wurden ${ }^{40}$, doch leider bleibt die Studie auf der Ebene der bivariaten Korrelationen stehen. Weder berücksichtigen die Autoren in der Auswertung des Zusammenhangs zwischen Mediennutzung und Schulleistung den sozial-kulturellen Hintergrund der Befragten, noch bedienen sie sich angemessener statistischer Verfahren, um den Einfluss der Nutzung einzelner Medien auf die schulische Leistung parallel zu erfassen. Zudem muss es als problematisch angesehen werden, dass Schülerinnen und Schüler einer Altersspanne von acht bis achtzehn Jahren untersucht wurden, ohne in der Auswertung zwischen einzelnen Altersgruppen zu differenzieren, obwohl die gegenwärtige Studienlage zu Fernsehnutzung und Schulleistung recht eindeutig zeigt, dass Mediennutzungseffekte auf die schulische Leistung eher bei jüngeren Altersgruppen zu erwarten sind (vgl. auch Mößle et al., 2007, S. 102).

Ausdrücklich hingewiesen werden muss bei allen zitierten Studien zur Wirkung der Computer(-spiel-)nutzung auf Schulleistung, dass es sich um Querschnittsstudien handelt, so dass kausale Wirkrichtungen zwar inhaltlich plausibel sind, empirisch aber nicht die gleiche Aussagekraft haben, wie die oben beschriebenen Längsschnittstudien zur Wirkung des Fernsehens auf Lese- und Schulleistungen. Weis und Cerankosky (2010) trugen diesem Defizit Rechnung und veröffentlichten eine feldexperimentelle Studie, in deren Rahmen sie den Spielkonsolenbesitz von Grundschulkindern systematisch variierten und die Effekte des Gerätebesitzes auf Fähigkeiten in Lesen, Mathematik und schriftlichem Ausdruck untersuchten. Im Rahmen dieser Studie wurden N = 64 Jungen zwischen sechs bis neun Jahren (Durchschnittsalter $=7.9$ Jahre) untersucht, wobei eine Teilgruppe der Jungen $(n=34)$ eine Spielkonsole erhielt ${ }^{41}$. Im

40 Die in dieser Studie ebenfalls betrachtete Nutzung von Fernsehen, DVD- und Videofilmen hatte aber keine signifikanten negativen Effekte auf die schulische Leistung, wenngleich auf der deskriptiven Ebene regelmäßige Nutzer dieser Medien leicht schlechtere Schulleistungen aufwiesen.

41 Voraussetzung für eine Teilnahme an der Studie war vorher bekundete Bereitschaft der Eltern der Jungen, ihren Kindern in absehbarer Zeit eine Spielkonsole kaufen zu wollen, wobei zu Beginn der Studie keines der Kinder eine Spielkonsole besaß. Jungen der 
Rahmen einer Pre- und einer Post-Messung (nach vier Monaten) wurden mithilfe standardisierter Tests die Lese,- Rechen,- und Schreibfähigkeiten der Jungen ermittelt. Zugleich wurden die Lehrkräfte der Kinder nach möglichen schulischen Problemen der Probanden befragt. Nach Kontrolle der Intelligenz der Jungen zeigten sich signifikante Unterschiede zwischen Kontroll- und Experimentalgruppe bezüglich der Lese- und Schreibfähigkeit nach Abschluss der Feldphase des Experimentes. Jungen der Experimentalgruppe schnitten in beiden Tests signifikant schlechter $a b$, in den Mathematiktests zeigten sich keine Unterschiede. Den Effekt auf die Lesefähigkeiten klassifizierten die Autoren mit $\eta^{2}=0.042$ als moderat, den Effekt auf den schriftlichen Ausdruck mit $\eta^{2}=0.081$ als groß. Zudem berichteten Lehrkräfte von Jungen aus der Experimentalgruppe in der Nachmessung signifikant häufiger von schulischen Problemen der Jungen. Als Mediator der Beziehung zwischen Spielkonsolenbesitz und den Leistungsproblemen der Jungen identifizierten die Forscher die Videospielzeiten der Jungen. Nach Kontrolle dieser Zeiten wurde die Beziehung zwischen Konsolenbesitz und Lese- beziehungsweise Schreibfähigkeiten nicht mehr statistisch bedeutsam. Aufgrund der Mediator-Funktion der Spielzeit interpretierten die Autoren die von Ihnen gefundenen Effekte als Beleg für die Zeitverdrängungshypothese. Als weitere Stütze für ihre Interpretation nannten sie die Tatsache, dass die von den Probanden genutzten Spieltitel nur auf moderate Gewaltexposition während der Feldzeit hindeuteten und das Treatment keine signifikanten Effekte auf das Sozialverhalten der Probanden hatte. Insofern sahen die Autoren für Wirkmechanismen in Sinne der Inhaltshypothese (vgl. S. 66 in dieser Arbeit) keine Belege.

\subsubsection{Fernseh- und Computerspielnutzung als Einflussfaktoren auf Schulleistung}

Einige neuere Studien haben versucht, Effekte der Fernseh- und Computerspielnutzung parallel $\mathrm{zu}$ messen und $\mathrm{zu}$ modellieren, um so dem Medienalltag von Schülerinnen und Schülern und daraus folgenden Effekten auf schulische Leistung eher gerecht zu werden. So berichten Mößle et al. (2007) im Rahmen ihrer Analyse von Daten der KFN-Grundschülerbefragung 2005 (zur Studien-

Kontrollgruppe bekamen die Spielkonsole nach Abschluss der Feldphase. Eine weitere Teilnahmevoraussetzung war, dass in der Biografie der teilnehmenden Jungen bisher keine Entwicklungs- oder Verhaltensstörungen aufgetreten waren und keine gesundheitlichen oder schulischen Probleme aufgetreten waren. Im Rahmen der Post-Messung wurde zudem sichergestellt, dass Jungen der Kontrollgruppe während der Feldzeit keine Spielkonsole bekommen hatten (Weis \& Cerankosky, 2010). 
anlage vgl. S. 29 in dieser Arbeit) von schwachen bis mittleren Effekten sowohl der Fernsehnutzung als auch der Nutzung von Computerspielen auf die schulische Leistung, wobei das Bildungsniveau im Elternhaus, das Familienklima und die Sprachkenntnisse der Kinder wie auch Ethnie und Alter der Kinder systematisch kontrolliert wurden. Vor allem konnte die Studie belegen, dass zeitlichen und inhaltlichen TV- und Computerspielnutzungsdimensionen unabhängig voneinander Einfluss auf die Schulleistung der befragten Schülerinnen und Schüler zukommt. Die Autoren kommen zu dem Schluss, dass sowohl auf der Verdrängungshypothese basierende Theorien wie auch die Inhaltshypothese bestätigt werden konnten ${ }^{42}$. Dabei kommt der Nutzung von Gewaltmedieninhalten mit entwicklungsgefährdendem Potential eine tendenziell höhere Wirkung zu als der rein zeitlichen elektronischen Mediennutzung. Das in Abbildung 1 gezeigte Strukturgleichungsmodell zeigt drüber hinaus, dass aufgrund der höheren Mediennutzungszeiten und der vergleichsweise häufig benutzten problematischen Medieninhalte vor allem Jungen unter Medieneffekten auf die schulische Leistung zu leiden haben. Ein darüber hinaus gehender Interaktionseffekt zwischen Geschlecht und Mediennutzung auf Schulleistung kann dagegen nicht festgestellt werden. Jungen und Mädchen mit vergleichbarer Mediennutzung und vergleichbarem sozio-kulturellen Hintergrund wiesen durchschnittlich nicht signifikant voneinander abweichende Schulnoten auf (Mößle et al., 2007, S. 102 - 103).

42 Allerdings weist Rehbein (2010) in seiner Darstellung der KFN-Ergebnisse nicht zu Unrecht darauf hin, dass bei den Mediennutzungszeiten zwischen Fernsehnutzungszeiten und Computerspielnutzungszeiten unterschieden werden sollte. Bei getrennten varianzanalytische Auswertungen zum Einfluss von Fernseh- bzw. Computerspielzeiten auf Schulleistungen zeigte sich für die Gruppe der durch ihre sonstigen Lebensumstände eher bevorzugten Schülerinnen und Schüler ein robuster, signifikanter Fernsehzeiteffekt, während Computerspielzeiten zwar ebenfalls in der Tendenz negativen Einfluss hatten, dieser jedoch knapp das Signifikanzniveau von $p<.05$ verfehlte (Mößle et al., 2007, S. 97). 


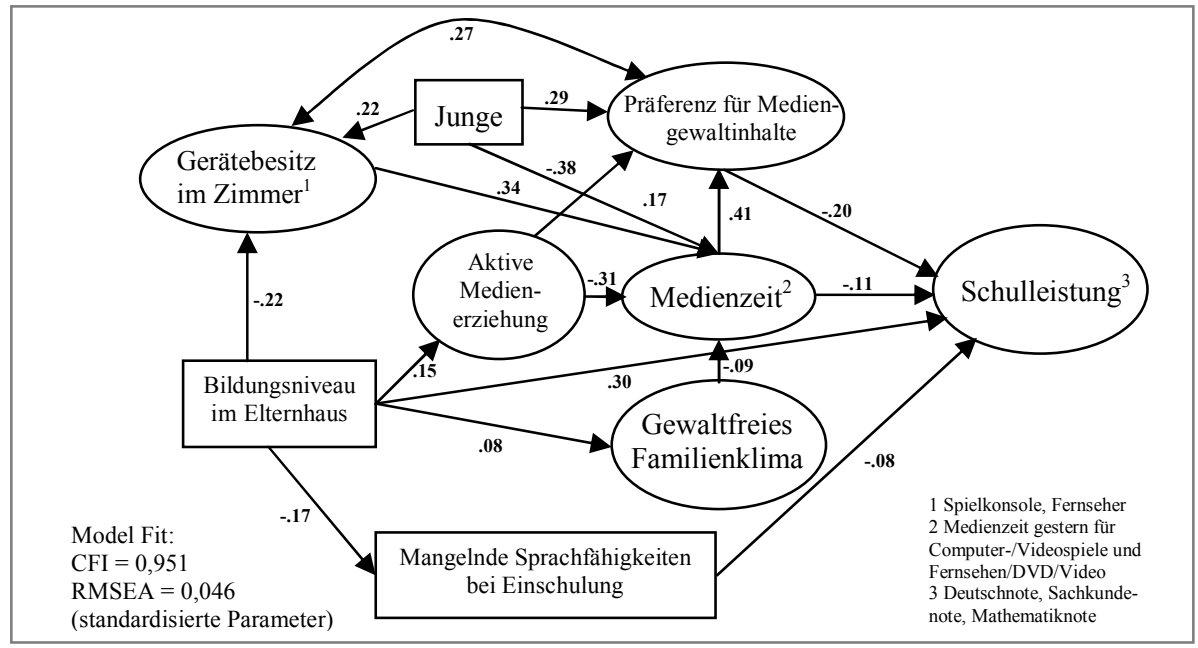

Abbildung 1: Pfadmodell zum Einfluss von Mediennutzung auf Schulleistung (entnommen aus Mößle et al., 2007, S. 99) ${ }^{43}$

Darüber hinaus zeigte die Studie, dass Medieneffekte auf Schulleistungen keineswegs nur in so genannten Risikopopulationen auftreten können. Zwar zeigt sich eindeutig, dass soziokulturell benachteiligte Kinder einem höheren Risiko unterliegen, ein eher problematisches Mediennutzungsverhalten aufzuweisen, gleichzeitig wurde aber deutlich, dass die Leistungen von Schülerinnen und Schüler mit vergleichsweise hohem soziokulturellen Status in besonderem Maße unter der Mediennutzung leiden, sollte diese durch lange Nutzungszeiten und problematische Medieninhalte gekennzeichnet sein (S. 130). Auf ähnliche internationale Befunde in Studien zum Effekt der Fernsehnutzung auf schulische Leistung weisen auch Comstock und Scharrer (1999, S. 248) in ihrem Review über die Resultate großer US-amerikanischer Schulleistungsstudien hin. Während sich bei durch ihre sonstigen Lebensumstände eher bevorzugten Schülerinnen und Schüler der stärkste Fernsehzeiteffekt auf Schulleistung zeigte, konnten stark benachteiligte Schülerinnen und Schüler durch lange Fernsehzeiten eher noch profitieren.

43 Weitere im Modell kontrollierte Faktoren: Ethnie, Alter der Kinder und Einfluss des Geschlechts auf Deutsch- und Mathematiknote. 
4.1.7 Fazit zum Zusammenhang zwischen elektronischer Mediennutzung und Schulleistung

Zusammenfassend lässt sich festhalten, dass die derzeitige Studienlage eindeutig negative Effekte der Nutzung elektronischer Bildschirmmedien auf die schulische Leistung von Kindern und Jugendlichen ergibt, wobei insbesondere Vor- und Grundschulkinder von solchen Effekten betroffen sind. Obwohl ausdrücklich darauf verwiesen werden muss, dass elektronische Mediennutzung nur ein (und selbstredend nicht der wichtigste) Einflussfaktor auf schulische Leistungen ist, zeigt doch die Forschungslage, dass sowohl hinsichtlich der Fernsehnutzung als auch der Computerspielnutzung eindeutige Belege für einen negativen Zusammenhang mit schulischer Leistung existierten. Dabei sprechen die Ergebnisse verschiedener Studien dafür, dass sowohl der Art der genutzten Medien als auch der für die Mediennutzung aufgewendeten Zeit ein Effekt für schulische Leistungsparameter zukommt. Solche Effekte treten zwar subgruppenspezifisch differenziert auf, andererseits lassen sich aber keine klaren Tendenzen beschreiben, welche Schülerinnen und welche Schüler als besonders gefährdet gelten. Keinesfalls sollte diese Ergebnislage dahingehend interpretiert werden, dass elektronische Medien per se einen leistungsmindernden Einfluss haben. So konnte die Forschung überzeugende Befunde zu leistungsfördernden Effekten einzelner Fernsehsendungen wie auch einzelner Computerspiele liefern. Solche Effekte zeigten sich aber ausschließlich bei auf die jeweilige Altersgruppe zugeschnittenen Medienangeboten mit klarem edukativem Hintergrund. Gleichzeitig muss aber darauf hingewiesen werden, dass die Nutzung solcher Medien im Alltag von Schülerinnen und Schülern kaum eine Rolle spielt und dass die von ihnen präferierten Medienangebote solche entwicklungs- und leistungsfördernden Effekte nicht haben. Zugleich zeigen bisherige Studien, dass es zwar bestimmte Risikopopulationen und Kindern und Jugendlichen gibt, die besonders dazu neigen, eine zeitlich oder inhaltlich problematische Mediennutzung aufzuweisen, trotzdem sind die Effekte einer solchen Mediennutzung bei allen soziokulturellen Subgruppen zu erkennen. 


\subsection{Mediennutzung und Sozialverhalten}

Wenn die Frage nach den Effekten der Medien auf das Sozialverhalten von Mediennutzern gestellt wird, steht dahinter zumeist nicht das Interesse an Medieneffekten auf das gesamte Sozialverhaltensrepertoire eines Menschen, sondern ein Interesse an bestimmten Teilen menschlichen Sozialverhaltens. Geht man davon aus, dass Sozialverhalten alle menschlichen Verhaltensweisen umfasst, die auf Reaktionen beziehungsweise Aktionen anderer Menschen zielen, wäre die Frage nach Medieneffekten tatsächlich nicht umfassend zu beantworten. Im Kontext der Debatte um Medieneffekte auf Kinder und Jugendliche wird unter Sozialverhalten zumeist ausdrückliches prosoziales Verhalten verstanden, also ein Verhalten, das ein einträchtiges Zusammenleben ermöglicht und fördert beziehungsweise sein genaues Gegenteil, das antisoziale Verhalten, welches in sozialen Konflikten mündet und diese zum Teil gezielt fördert. In diesem Sinne sollen auch im folgenden Abschnitt nicht Medieneffekte auf die Gesamtheit menschlichen Sozialverhaltens dargestellt werden, sondern lediglich solche Effekte auf explizit antisoziales und prosoziales Verhalten.

\subsubsection{Antisoziales Verhalten}

\subsubsection{Theoretische Modelle}

Nach Bandura sind Menschen - von elementaren Reflexen abgesehen - nicht mit angeborenen Verhaltensrepertoires ausgestattet, so dass diese erlernt werden müssen (Bandura, 1979, S. 25). Sozialverhalten wird dabei wie andere Verhaltensweisen auch vielfach durch unmittelbares Erfahrungslernen vermittelt (S. 26), indem sich Menschen mit positiven und negativen Wirkungen ihrer Handlungen auseinandersetzen und ihr weiteres Handeln danach ausrichten, welche Handlungen als erfolgreich beurteilt wurden (Bekräftigungslernen) (ebenda). Bandura verweist darauf, dass Lernen "ein außerordentlich mühsames Geschäft [wäre] - vom Risiko ganz zu schweigen - wenn die Menschen als einzige Richtlinie für künftiges Tun nur die Auswirkungen ihres eigenen Handelns hätten" (S. 31). So werden nach Bandura die meisten menschlichen Verhaltensweisen durch Beobachtung von Modellen erlernt und dem Lernenden bleiben so, bevor er die betreffende Verhaltensweise selbst ausgeführt hat, überflüssige Fehler erspart (ebenda). Empirische Belege für Banduras Theorie vom Lernen am Modell wurden frühzeitig am Beispiel der Imitation filmisch vermittelter aggressiver Verhaltensmuster durch Kinder experimentell bestätigt (Bandura, 
Ross \& Ross, 1961, 1963) und wurden seitdem fester Bestandteil des Kanons klassischer Gewaltmedienwirkungstheorien (vgl. etwa Gleich, 2004; HoppeGraff \& Kim, 2002; Kunczik, M. \& Zipfel, 2004; Petersen \& Kleimann, 2005). Dies hatte zur Folge, dass Banduras sozial-kognitive Lerntheorie (Bandura, 1979) in der medienwissenschaftlichen Debatte fortwährend im Kontext der Gewaltmediendebatte wahrgenommen wurde, während Gentile et al. (2009, S. 753) zu Recht darauf hinweisen, dass der dem Modell-Lernen zugrundeliegende lerntheoretische Ansatz Ausgangspunkt für eine deutlich umfassendere Theorie zum Verständnis von Mediennutzung und ihren (negativen wie positiven) Wirkungen sein kann.

Anderson und Bushman (Anderson, C. A. \& Bushman, 2002; Bushman \& Anderson, 2002) haben verschiedene Theorien ${ }^{44}$ kurzfristiger und langfristiger, kognitiver und emotionaler Gewaltmedienwirkungen zusammengetragen und mit ihrem General Aggression Model ein umfassendes lerntheoretisches Modell kurzfristiger und langfristiger Gewaltmedienwirkungen formuliert. Im Rahmen dieses Modells zeigen die Autoren, dass die Wirkung gewalthaltiger Medieninhalte in maßgeblicher Weise von situativen Eigenschaften der Nutzung und personalen Eigenschaften des Nutzers abhängt, die in entscheidender Weise die Verarbeitung gewaltmedialer Stimuli und eventuelle Verhaltenseffekte oder Effekte in der Ausbildung bestimmter Erregungszustände sowie kognitiver und affektiver Strukturen beeinflussen. Jeder einzelne Gewaltmediennutzungsvorgang wird dabei als Lernprozess verstanden, wobei zwischen (zum Teil unbewussten) Lernvorgängen niedriger Ordnung (etwa assoziatives Lernen durch Verknüpfen positiver oder negativer Affekte mit bestimmten audiovisuellen Inputs $^{45}$ ) und elaborierten Lernvorgängen (zum Beispiel dem Modell-Lernen zum Verhalten in bestimmten sozialen Konfliktsituationen) unterschieden werden kann. Als Resultat solcher Lernprozesse entsteht nach Anderson und Bushman langfristig ein Netzwerk bestimmter Wahrnehmungsskripte und -schemata sowie

44 Die Autoren verknüpfen (S. 29 - 32) in ihrem General Aggression Model die Cognitive Neoassociation Theory (Collins \& Loftus, 1975), Sozial-kognitive Lerntheorie (Bandura, 1979), Skript-Theorie (Schank \& Abelson, 1977), Excitation-Transfer-Theorie (Zillmann, 1983) und Social Interaction Theory (Tedeschi \& Felson, 1994).

45 Der Anblick eines Messers kann demnach je nach Vorerfahrungen ein Assoziationsnetz aggressiver Konzepte, Angst-/Fluchtkonzepte und selbst Harmoniekonzepte aktivieren, je nachdem ob der Beobachter mit einem solchen Gegenstand positiv-aggressive Erfahrungen (etwa durch den effektiven Messereinsatz in einem Computerspiel), negative Erfahrungen (als „Beobachter“ einer brutalen Raubüberfall-Szene in einem Roman) oder positiv harmonische Erfahrungen (durch die Aktivierung eines im Fernsehen gesehenen Konzeptes „Messer als hochwertiges Werkzeug zur Herstellung einer Holzschnitzerei“) gemacht hat. 
entsprechender Verhaltensskripte und -schemata, die etwa in sozialen Konfliktsituationen aktiviert werden.

In der Folge kann sich bereits die Wahrnehmung einer nicht sofort eindeutig $\mathrm{zu}$ interpretierenden sozialen Situation stark zwischen zwei Individuen unterscheiden. So kann ein Zusammenstoß mit einem Passanten auf der Straße entweder als unglücklicher Zufall oder als absichtliche Handlung des Anderen interpretiert werden (Theorie und empirische Befunde zum so genannten hostile attribution bias etwa bei Crick \& Dodge, 1994). Selbst bei einheitlicher Wahrnehmung und Interpretation der Situation als Unfall unterscheiden sich unter Umständen die Verhaltensresultate interindividuell sehr deutlich ${ }^{46}$. Und auch die Reaktion des Anderen kann unterschiedlich interpretiert werden ${ }^{47}$. Werden die eben beschriebenen Wahrnehmungs- und Entscheidungsprozesse durch die fortwährende Rezeption medialer Gewalt beeinflusst und geprägt, fördert dies nach Anderson und Bushman die langfristige Ausbildung einer aggressiven Persönlichkeit (Bushman \& Anderson, 2002, S. 1680).

Die Extremform antisozialen Verhaltens bildet ohne Zweifel das Gewaltverhalten beziehungsweise das gewalttätige Verhalten. Im Rahmen der Gewaltmedienwirkungsforschung muss zunächst deutlich unterschiedenen werden, welche Gewalt im Fokus des Forschungsinteresses steht: Die in den Medien präsentierte Gewalt, die aus medialen Nutzungsangeboten resultierende Gewalt, oder beides zugleich. Im Rahmen medien-, kommunikations,- oder publizistikwissenschaftlicher Arbeiten wird die Definition von Gewalt in der Regel anhand der medial präsentierten Gewalt vorgenommen. Nach Merten (1999, S. 62) lässt sich Gewalt im engeren Sinne als "zielgerichtete, direkte Schädigung von Menschen durch Menschen beziehungsweise als körperlicher Angriff auf Sachen verstehen". Menschliches Gewaltverhalten kann nach Bushman und Anderson (2001), Geen (2001), Baron und Richardson (1994) sowie Berkowitz (1993) definiert werden als

46 Entschuldige ich mich als erster, um die Situation - unabhängig von meiner tatsächlichen Schuld - zu bereinigen, so wie ich es durch Beobachtungen in den Medien oder der Realität gelernt habe? Erwarte ich zuerst die Entschuldigung des Anderen - weil er jünger, kleiner oder neu in der Gegend ist? Stelle ich den Anderen analog zum Verhalten meiner realen oder medialen Vorbilder sofort zur Rede, da ich seine Unaufmerksamkeit als Respektlosigkeit und Ehrverletzung betrachte und ein solches Verhalten mir gegenüber nicht dulden kann?

47 War das Abwinken des Anderen als Geste des Eingestehens eigener Schuld zu verstehen oder als weiteres Zeichen der Ignoranz, so dass ich in einer ähnlichen Situation ähnlich wie meine medialen oder realen Vorbilder zukünftig noch bestimmter oder aggressiver auftreten sollte, um eine angemessene Reaktion zu erhalten? 
"...any behavior directed toward another individual that is carried out with the proximate (immediate) intent to cause harm. In addition, the perpetrator must believe that the behavior will harm the target, and that the target is motivated to avoid the behaviour." (hier zitiert nach Anderson, C. A. \& Bushman, 2002, S. 28).

Diese Definition enthält bereits diverse Einschränkungen ${ }^{48}$ des Gewaltbegriffs, erscheint aber dem ganz überwiegenden Teil der Gewaltmedienforschung angemessen, wobei sich im Rahmen der Medieninhaltsforschung trotz des recht engen Gewaltbegriffs zahlreiche Probleme in der Messung solcher Gewalt ergeben $^{49}$. Im Rahmen dieser Arbeit, die in diesem Abschnitt nach Medienwirkungen auf das Verhalten (von Kindern und Jugendlichen) fragt, interessiert aber vor allem die aus medialen Nutzungsangeboten resultierende Gewalt beim (minderjährigen) Nutzer. Die Mehrheit der einschlägigen Studien wählt hier (allein schon aus methodischen Gründen) zumeist nicht gewalttätiges Verhalten als resultierende Variable, sondern verschiedene Formen aggressiver Einstellungen, Kognitionen und Verhaltensäußerungen, also lediglich gewaltnaher Parameter. Der noch wenig überzeugenden Aufteilung solcher Parameter von Paik und Comstock (1994) in ihrer Meta-Analyse der Wirkungen von Fernsehgewaltnutzung $^{50}$ setzen Anderson und Bushman in ihren Meta-Analysen zur Wirkung von interaktiver (Computer-)Mediengewalt die folgende Aufteilung

48 So subsumiert Merten (1999, S. 21 - 22) zunächst völlig zu Recht auch strukturelle Gewalt, kollektive Gewalt, latente Gewalt und nicht-intentionale Gewalt unter einem breiten Gewaltbegriff, verweist aber zugleich auf die Schwierigkeiten, die sich aus der Unschärfe eines derart breiten Gewaltbegriffes ergeben, etwa hinsichtlich der Objektivierbarkeit dieser Arten von Gewalt und der damit verbundenen Schwierigkeiten einer Operationalisierbarkeit von Gewalt im Rahmen empirischer, insbesondere inhaltsanalytischer Studien.

49 Etwa in Bezug auf die Zielgerichtetheit/Intentionalität einer Handlung oder der dem Willen des Opfers, nicht Ziel von Gewalt zu werden. Anderson und Bushman (2002, S. 29) verweisen auf das Beispiel des freiwilligen, obschon schmerzhaften Zahnarztbesuchs sowie auf sado-masochistische sexuelle Praktiken.

50 Paik und Comstock (1994, S. 531 - 533) unterscheiden bei den Wirkungen von Fernsehgewalt zwischen simuliert-aggressivem Verhalten (simulated aggressive behavior), geringfügig aggressivem Verhalten (minor aggressive behavior) und illegalen Handlungen (illegal activites). Unter simuliert-aggressivem Verhalten werden dabei aggressive Verhaltensmuster von Probanden in experimentellen Studien verstanden, die beispielsweise vorgeblich echte Elektroschockgeräte bei anderen Probanden auslösen oder mit Gewaltspielzeug spielen. Diese Formen simuliert-aggressiver Handlungen setzen Paik und Comstock selbstberichteter Gewaltintention gleich. Als geringfügig aggressiv bezeichnen die Autoren Gewalthandlungen gegen Sachen oder Gewalt gegen Personen, die nicht als illegal gesehen werden kann. Unter den illegalen (Gewalt-)handlungen verstehen Paik und Comstock Diebstahl (burglary), schweren Diebstahl (grand theft) sowie jegliche direkte illegale Gewalt gegen Personen (Mord, Selbstmord, Messerstecherei, etc.). 
gewaltnaher Parameter entgegen: Sie unterschieden zunächst zwischen aggressivem Verhalten (aggressive behavior), aggressiven Kognitionen (aggressive cognitions), aggressiven Affekten (aggressive affects), Unterstützungsverhalten in aggressiven Konfliktsituationen (helping behavior) und allgemeiner körperlicher Erregung (physiological arousal) (Anderson, C. A., 2004; Anderson, C. A. \& Bushman, 2001). Diese an der zumeist kurzfristigen Messbarkeit aggressionsnaher Parameter ausgerichtete Aufteilung ergänzten sie später im Rahmen ihres General-Aggression-Models um die folgende Aufteilung, in der auch längerfristige Gewaltmedienwirkungen berücksichtigt werden (vgl. Anderson, C. A. \& Bushman, 2002, S. 42): Aggressive Überzeugungen und Einstellungen (aggressive beliefs and attitudes), aggressive Wahrnehmungsschemata (aggressive perceptual schemata), aggressive Erwartungsschemata (aggressive expectation schemata), aggressive Verhaltensskripte (aggressive behavior scripts) sowie Desensibilisierung für Aggressivität (aggressive desensitization). Eine Kumulation all dieser Faktoren führt nach Anderson und Bushman langfristig zur Ausbildung und Festigung einer aggressiven Persönlichkeit (increase in aggressive personality).

Der von Anderson und Bushman vorgeschlagenen letzten Kategorie von Gewaltmedienwirkungen, der Aggressions- beziehungsweise Gewaltdesensibilisierung kommt hier eine Sonderrolle zu. Denn die Desensibilisierungsforschung proklamiert zunächst keine Steigerung von Aggressivität, sondern eine allmähliche Abschwächung beziehungsweise Löschung kognitiver, emotionaler und verhaltensbezogener Reaktionen auf Mediengewalt infolge wiederholter Gewaltmediennutzung (Mößle et al., 2007, S. 36). Dieser empirisch inzwischen gut belegte Prozess (Bartholow, Bushman \& Sestir, 2006; Carnagey, Anderson \& Bushman, 2007; Funk, Baldacci, Pasold \& Baumgardner, 2004; eine Kurzübersicht zur Desensibilisierungsforschung bei Mößle \& Kleimann, 2009) stellt aber keineswegs einen Widerspruch zur sonstigen Mediengewaltforschung dar. Vielmehr wird als Folge von Gewaltdesensibilisierung proklamiert, dass einerseits die Sensibilität für reale Gewaltkonflikte geschwächt wird, was zu geringerer Hilfs- oder Eingriffsbereitschaft bei realen gewalttätigen Konflikten führen kann, und dass andererseits die Hemmschwelle zur eigenen Anwendung von Gewalt sinkt, da entsprechende Situationen im Rahmen von Filmmedienrezeption oft genug stellvertretend durch den Protagonisten miterlebt oder im Rahmen eines Computerspiels selbst trainiert wurden. Carnagey et al. resümieren: In short, the modern entertainment medial landscape could accurately be described as an effective systematic violence desensitization tool." (Carnagey et al., 2007, S. 7). 
Obwohl die Erforschung der Wirkungen von Mediengewalt fortwährend von methodologischen Debatten (etwa um die Eignung korrelativer Querschnittsuntersuchungen zur Aussage über Medienwirkungen) und (durchaus notwendigen) Diskussionen um definitorische und theoretische Fragen begleitet wurde, ergibt sich zum aktuellen Zeitpunkt ein zwar differenziertes, tendenziell jedoch recht eindeutiges Bild ${ }^{51}$. So kommen Huston und Wright 1998 nach einem Review der Studienlage zur Wirkung von Fernsehgewalt auf Kinder zu dem folgenden Schluss: „There is little doubt that viewing television violence has a causal effect on aggressive behavior, though there is disagreement about the magnitude and social importance of the effect.“ (Huston \& Wright, 1998, S. 1043). Noch eindeutiger äußert sich Huesmann in seinem Review zum Fortschritt von empirischer Forschung und Theorielage der Gewaltmedienforschung:

"Since the early 1960s, research evidence has been accumulating that suggests that exposure to violence in television, movies, video games, cell phones, and on the Internet increases the risk of violent behavior on the viewer's part, just as growing up in an environment filled with real violence increases the risk of them behaving violently." (Huesmann, L. R., 2007, S. 6)

Michael Kunczik und Astrid Zipfel, die 2004 die letzte große Aktualisierung der in Deutschland einschlägigsten Zusammenfassung der Gewaltmedienforschung vorgelegt haben kommen zu dem folgenden Fazit:

"Versucht man trotz der erwähnten vielfältigen Probleme eine Bilanz zu ziehen, so ist zunächst festzuhalten, dass die Annahme einer generellen Ungefährlichkeit von Mediengewalt fast nicht mehr vertreten wird. Weitreichende Übereinstimmung herrscht auch darüber, dass die Auswirkungen von Mediengewalt jedoch differenziert betrachtet werden müssen. Die Korrelationsmaße der meisten Untersuchungen sprechen für einen kleinen bis mittelstarken Zusammenhang zwischen Mediengewalt und Aggressivität des Rezipienten.“ (Kunczik, M. \& Zipfel, 2004, S. 289)

51 Dass diese Tatsache gar nicht oft genug betont werden kann, zeigt eine Untersuchung zur Differenz zwischen wissenschaftlicher Erkenntnislage und der nachrichtenmedialen Darstellung des Zusammenhangs zwischen Mediengewalt und Gewaltverhalten in Amerika (Bushman, Brad J. \& Anderson, Craig A., 2001). Die Autoren zeigen, dass seit Anfang der 1970er-Jahre bis Anfang des 21. Jahrhunderts in der medialen Berichterstattung die Wirkung von Mediengewalt auf Gewaltverhalten zunehmend relativiert wurde, während die internationale Studienlage zur Gewaltmedienforschung seit Anfang der 1990er-Jahre eher höhere Korrelationen zwischen Mediengewalt und Gewaltverhalten ausweist, als noch in den 1970er- und 1980-Jahren. 


\subsection{Meta-Analysen zur TV-Gewalt}

Die in den oben zitierten Reviews zitierten Aussagen werden durch eine Reihe von Meta-Analysen gestützt, die in den letzten Jahren veröffentlicht wurden. So resümieren Paik und Comstock (1994) in ihrer Meta-Analyse von 217 empirischen $^{52}$ Studien zur Wirkung von TV-Gewalt von 1957 bis 1990, dass Fernsehgewalt aggressives und antisoziales Verhalten eindeutig erhöhe (der mittlere Zusammenhang zwischen Fernsehgewalt und Gewaltverhalten lag demnach bei $r=0.31$ und Cohen's $d=0.65$ ), wobei die Stärke des Effektes vor allem abhängig von der betrachteten zu erklärenden Variable sei (S. 538). So zeigten sich für explizite Gewaltkriminalität die geringsten Effektstärken der Fernsehgewaltnutzung, für niederschwelligeres Gewaltverhalten zeigten sich höhere Effektstärken (ebenda). Paik und Comstock verweisen darauf, dass sich in den von ihnen analysierten Studien Effekte der Fernsehgewalt in allen Altersgruppen zeigten, sowohl in Vorschul- und Schulpopulationen, als auch bei Studierenden und Erwachsenen, wobei sich für die jüngeren Populationen die höheren Effektstärken zeigten, für die erwachsenen Populationen eher geringe Effektstärken (S. 537). Dies könne aber, so die Autoren, keinesfalls als Entwarnung verstanden werden (etwa insofern, als Vorschul- und Schulkinder am wenigsten in der Lage sein, aggressives Verhalten in einer für die Gesellschaft gefährlichen Form auszuleben). Stattdessen verweisen Paik und Comstock auf die durch Längsschnittstudien belegten Langzeitwirkungen früher Gewaltmediennutzung auf Gewaltverhalten im Erwachsenenalter (ebenda) ${ }^{53}$.

52 In die Analyse einbezogen wurden labor- und feldexperimentelle Studien, Längsschnittanalysen sowie Fragebogenuntersuchungen im Querschnittsdesign.

53 Die von Paik und Comstock als Beleg zitierten Panelstudien von Lefkowitz, Eron, Walder und Huesmann (1977) sowie von Milavsky, Kessler, Stipp und Rubens (1982) wurden allerdings insbesondere von Savage (2004) in verschiedenen datenanalytischen Aspekten sowie der Operationalisierung von Gewaltverhalten als abhängiger Variable scharf kritisiert. In einer Meta-Analyse der bisherigen Forschung zur Wirkung von Medien-, und insbesondere Fernsehgewalt kommen Savage und Yancey (2008) sogar zu dem Schluss, sie hätten keine einzige Studie finden können, die ihren Ansprüchen an die Erforschung des Zusammenhangs von Mediengewalt und Gewaltverhalten genügen könne (S. 786). Es erscheint allerdings befremdlich, dass die Autoren auf Grundlage dieser Argumentation folgern, die 26 von ihnen analysierten Studien ,did not suggest that exposure to media violence is associated with criminal aggression" (Savage \& Yancey, 2008, S. 772), obwohl 15 dieser Studien signifikante Mediengewalteffekte berichten. Stattdessen erklären die Autoren: "In summary, because the burden, in science, is to reject the null hypothesis only when we have a high degree of confidence that actual values 
In einer weiteren Studie unterzog Comstock (2008) 14 Jahre nach seiner Meta-Analyse zum Zusammenhang zwischen Fernsehnutzung und Gewaltverhalten sieben meta-analytische Studien $^{54}$, die den Zusammenhang zwischen Film- und Fernsehnutzung beziehungsweise Film- und Fernsehgewaltdarstellungen und Gewaltverhalten untersuchen, einem detaillierten Review (darunter die eigene Studie von 1994). Auch im Rahmen dieser Studie zeigte sich in allen meta-analytischen Studien ein signifikanter Zusammenhang zwischen genutzten Gewaltinhalten in Film- und Fernsehen und aggressivem Verhalten (S. 1187), wobei Paik und Comstocks eigene Studie den größten Effekt fand $(r=0.31)$ und eine Meta-Analyse von Hogben (1998) mit $r=0.11$ den geringsten Effekt. Obwohl Comstock bei Sichtung aller Meta-analytischen Studien, ähnlich wie in der Meta-Analyse von 1994, die größten Zusammenhänge zwischen Fernsehnutzung und Gewalt bei sehr jungen (Vorschulkindern) fand, zeigten Studien mit älteren Probanden keine wesentlich kleineren Effekte der Gewaltmediennutzung. Diesen im Widerspruch zur Studie von 1994 stehenden Befund interpretiert er so, dass offenbar die Mediengewalteffekte nicht automatisch kleiner werden, wenn mit zunehmenden Alter die kognitiven Fähigkeiten zur Fiktions-Realitäts-Unterscheidung und zur Differenzierung zwischen mitreißenden Szenen und den unter Umständen zwielichtigen Motiven der Protagonisten wachsen. Stattdessen seien, so seine Interpretation, die von Jugendlichen und Erwachsenen genutzten Gewaltdarstellungen deutlich komplexer und somit nicht mehr so eindeutig im Sinne einer Distanzierung von der gezeigten Gewalt zu interpretieren (S. 1196).

Comstock identifiziert in den von ihm analysierten Studien fünf Faktoren, die Fernsehgewalteffekte auf Aggressivität besonders zu begünstigen scheinen: Eine Prädisposition des Nutzers für aggressives beziehungsweise antisoziales Ver-

are not zero, using the most rigorous methods available before drawing firm conclusions about a phenomenon, it is our conclusion that the effects of exposure to media violence on criminally violent behavior have not been established" (S. 787). Dieser Argumentation kann mit Comstock (2008) entgegnet werden, der unter Berufung auf Hunter und Smith (2004) bemerkt: "the perfect study is a myth, but the weaknesses of any one or several studies - in operationalization, sample, or data analysis and interpretation - are largely overcome when studies are pooled for analysis." (S. 1188).

54 Eine Liste der untersuchten Meta-Analysen bei Comstock (2008, S. 1187). Entgegen Comstocks Beschreibung (S. 1186) der Meta-Analyse von Bushman und Anderson (2001) bezieht sich letztere aber nicht ausschließlich auf Fernsehgewalt, sondern auf sämtliche experimentellen Studien bezüglich medialer Gewalt und Aggressivität. Nach Bushman und Anderson wurde die PsychINFO-Datenbank zu diesem Zweck nach den folgenden Begriffen durchsucht: violen* oder aggress*, TV, televis*, film, movie, screen, music, radio, video game, computer game, electronic game, cartoon, comic, pornograph*, erotic*, news, book, magazine, sport (S. 484). 
halten, eine zu strenge oder gleichgültige elterliche Erziehung, unbefriedigende soziale Beziehungen, niedrige psychische Gesundheit oder eine diagnostizierte Verhaltensstörung (S. 1205). Insgesamt, so Comstock, werde die Hypothese zum Zusammenhang zwischen Fernsehnutzung und aggressivem sowie antisozialen Verhalten durch seine Analyse bestätigt. Die von ihm betrachteten MetaAnalysen sprächen des Weiteren für einen kausalen Zusammenhang von Gewaltmediennutzung und Gewalt (S. 1184). Mit Blick auf die mit allgemein prekären Entwicklungsbedingungen (sozioökonomischer Status, elterliche Bildung, Zugang zu Bildungschancen, etc.) von Kindern verknüpften fünf Begünstigungsfaktoren der Effekte von Mediengewalt, kommt Comstock zu dem folgenden Schluss:

"This raises questions about the social function of the media and their possible rolealthough surely unintended by anyone who has anything at all to do with them - in maintaining an underclass of the marginally educated, marginally employed, and frequently incarcerated." (Comstock, G., 2008, S. 1207).

Auch Christensen und Wood (2007) kamen in ihrer Meta-Analyse zu dem Ergebnis, dass Fernsehgewalteffekte auf Gewaltverhalten keinesfalls mit zunehmendem Alter linear abnehmen. Die Autoren analysierten ausschließlich experimentelle Studien mit Kindern und Jugendlichen, in denen das Gewaltverhalten der Probanden in der natürlichen Interaktion mit anderen Probanden im Anschluss an die Rezeption gewalthaltiger Fernsehinhalte durch geschulte Beobachter einzeln klassifiziert wurde. Als Gewaltverhalten wurde dabei direkte Gewalt gegen andere im Raum vorhandene Personen definiert (S. 152). Unter den 29 von ihnen gefunden zwischen 1950 und 2004 durchgeführten experimentellen Studien fanden Christensen und Wood 13 Studien, in denen Effektstärken berechnet werden konnten (S. 157). Als mittlere am jeweiligen Studiensample gewichtete Effektstärke dieser Studien berichten die Autoren ein $r=0.17$ beziehungsweise Cohens $d=0.35$ (S. 158). Der im Vergleich zu Comstocks Daten kleinere Fernsehgewalteffekt erscheint insofern plausibel, als die Messung der abhängigen Variable (direkt beobachtbare Gewalt gegen eine andere reale Person im Rahmen natürlicher Interaktion) als sehr konservatives $\mathrm{Maß}$ zu bezeichnen ist.

Bemerkenswert erscheint aber vor allem der zweite zentrale Befund der Studie, nach dem die Stärke des Zusammenhangs zwischen Fernsehgewalt und Gewaltverhalten zunächst zwischen Vorschulalter und spätem Grundschulalter 
ansteigt und in der Phase der Adoleszenz wieder abnimmt (S. 163) ${ }^{55}$. Die Autoren interpretieren dies als Bestätigung ihrer Annahme, dass sehr junge Kinder einerseits noch nicht in der Lage sind, mediale Gewaltbotschaften zu erfassen und aufzunehmen, so dass sie - zumindest nach recht kurzfristigem Stimulus - nicht zu direkter Gewalt gegenüber Gleichaltrigen führt, während andererseits Jugendliche aufgrund von Vorerfahrungen und bereits relativ klar herausgebildeten Einstellungen gegenüber Gewalt für experimentellen Mediengewaltstimuli nicht mehr so anfällig sind wie jüngere Kinder. Vor diesem Hintergrund postulieren die Autoren für das Verhältnis von Gewaltmedienanfälligkeit und Alter die Form einer umgekehrten U-Kurve, die im Grundschulalter ihren Höhepunkt hat, in einem Alter nämlich, in dem die kognitive Fähigkeit von Kindern zur Aufnahme medialer Botschaften bereits stark entwickelt ist, während die Internalisierung gesellschaftlicher gewaltbezogener Normen noch nicht komplett vollzogen ist und auch die Übernahme medialer Verhaltensmuster relativ unvoreingenommen geschieht.

\subsection{Meta-Analysen zur Computerspielgewalt}

Eine erste Meta-Analyse zum Zusammenhang zwischen der Nutzung von Computer- und Videospielen und Aggressivität wurde im Jahr 2001 von John Sherry vorgelegt. Darin untersuchte der Autor 25 voneinander unabhängige Studien mit einer Gesamtzahl von N = 2.722 Probanden, die bis zum Jahr 2000 zu diesem Komplex vorlagen (Sherry, S. 415). Dabei handelte es sich um sechs Querschnittsbefragungen und 16 Experimentalstudien, in denen Aggressivität beziehungsweise Gewaltverhalten entweder direkt (in Form von Verhaltensbeobachtungen oder Verhaltenstests) oder indirekt (via Fragebogen) gemessen wurde (S. 416 - 417). Mit einer mittleren Effektstärke von $r=0.15$ beziehungsweise Cohen's $d=0.30$ zeigte sich ein robuster, wenn auch schwacher $\mathrm{Zu}$ sammenhang zwischen der Nutzung gewalthaltiger Computerspiele und Aggressivität $^{56}$. Als verblüffend bezeichnet Sherry das Ergebnis seiner Studie, dass der Effekt der Gewaltspielnutzung auf Aggressivität bei Experimentalpro-

55 Für die Altersgruppe unter 6 Jahren berichten die Autoren einen Effekt von $d=0.27$ $(r=0.13)$, für die Altersgruppe zwischen 6 und 10 Jahren einen Effekt von $d=0.66$ $(r=0.31)$ und für jugendliche Probanden einen Effekt von $d=0.12(r=0.06)$.

56 In einer neueren Publikation relativiert Sherry (2007) dieses Ergebnis allerdings deutlich, indem er bemerkt, ein kausaler Zusammenhang zwischen beiden Variablen sei aufgrund seiner Meta-Analyse nicht zu ziehen, allenfalls wiese seine Studie auf eine erhöhte Wahrscheinlichkeit eines solchen Zusammenhangs hin (S. 259). 
banden negativ mit der Spielzeit korreliert war (S. 422 - 425). Sherry weist in diesem Zusammenhang darauf hin, dass der gefundene Zeiteffekt besonders auf zwei Studien beruhe, deren sonstige Parameter (Probandengruppe, gespieltes Spiel: Mortal Kombat, Operationalisierung der abhängigen Variable) gleich waren, so dass die unterschiedlichen Effekte der Studien mit hoher Wahrscheinlichkeit auf die Spielzeit zurückzuführen seien (S. 425). Möglicherweise, folgert der Autor, sei nach einer nur kurzen Spielzeit von 10 Minuten das allgemeine Arousal-Level der Probanden einfach höher, während nach einer Spielzeit von 75 Minuten eine Gewöhnung an das Spiel und die Spielgewalt stattgefunden habe und einige Probanden unter Umständen eher gelangweilt gewesen seien (ebenda). Vor diesem Hintergrund empfiehlt er weiterführende Studien, in denen Effekte von Desensibilisierung, Langeweile oder möglicherweise Effekte im Sinne der Katharsis-Hypothese untersucht werden (ebenda) ${ }^{57}$.

Ein Vergleich seiner Ergebnisse mit der Meta-Analyse von Paik und Comstock (1994) zum Zusammenhang zwischen Fernsehnutzung und Aggressivität, deren mittlere Effektstärke bei Cohen's $d=0.65$ lag, lässt den Autor dabei zunächst zu dem Schluss kommen, die Effekte der Nutzung interaktiver Gewalt auf Gewaltverhalten beziehungsweise Aggressivität seien kleiner als beim Fernsehen (Sherry, 2001, S. 424). Diese Interpretation kann aber durchaus kritisiert werden, wobei Sherry eine wichtige Überlegung selbst präsentiert. So berichtet er einen signifikanten Zusammenhang zwischen der in einer Studie gefundenen Effektstärke und dem Zeitpunkt ihrer Durchführung (S. 422). Je jünger die Studie war, desto größer war der berichtete Effekt. Sherry interpretiert dies so, dass einerseits der grafische Realismus der Spiele im Laufe der Zeit größer geworden sei und sich andererseits auch die Art der in den publizierten Studien genutzten Spiele mit der Zeit geändert habe. Handelte es sich bei den ersten Spielen häufig um recht abstrakte Spiele mit dem Hintergrund eines sportlichen Wettkampfes, sei die Art der präsentierten Gewalt in jüngeren Studien eher menschlich und realistisch gewesen (S. 424). Vor diesem Hintergrund lassen sich zukünftig möglicherweise größere Effekte der Computerspielnutzung auf Gewaltverhalten erwarten. Ein anderer Punkt, der Sherrys Interpretation in Frage stellt, Gewaltfernsehnutzung sei stärker mit Gewaltverhalten korreliert als die Nutzung von Gewaltcomputerspielen, ist die Frage danach, warum Sherry ausgerechnet die Analyse von Paik und Comstock (1994) als Referenzpunkt wählt, während vier der acht von Comstock (2008) identifizierten MetaAnalysen zu Fernsehgewalt und Gewaltverhalten Effektgrößen ausweisen, die kleiner oder gleich dem von Sherry berichteten Effekt von $r=0.15$ sind. 
Anderson, Bushman und Kollegen haben wiederholt den Zusammenhang zwischen der Nutzung interaktiver Computerspiele und verschiedener gewaltnaher Parameter (vgl. S. 86 in dieser Arbeit) meta-analytisch untersucht (Anderson, C. A., 2004; Anderson, C. A. \& Bushman, 2001; Anderson, C. A. et al., 2010). Nach Auswertung von insgesamt 136 Forschungsberichten experimenteller, längs- und querschnittlicher Studien mit 381 voneinander unabhängigen Stichproben kommen Anderson et al. (2010) zu dem Ergebnis, die Nutzung gewalthaltiger Computerspiele sei signifikant mit aggressivem Verhalten, vermindertem prosozialen Verhalten, gesteigerten aggressiven Kognitionen, gesteigerten aggressiven Affekten und gesteigerter physiologischer Erregung korreliert, sowie mit geringer Empathie und gesteigerter Gewaltdesensibilisierung. Allein für aggressives Verhalten als Folge der Nutzung von Computerspielgewalt berichtet Anderson von Messungen an 68.131 Probanden aus 140 unabhängigen Stichproben mit einer durchschnittlichen Effektstärke von $r=.19$ (Anderson, C. A. et al., 2010, S. 162). Damit lag der mittlere Effekt der Nutzung interaktiver Gewaltmediennutzung auch in diesen Studien etwas unter dem des Fernsehens. In ihrer jüngsten Meta-Analyse berücksichtigen Anderson et al. dabei auch zahlreiche mögliche Moderatoreffekte, wobei sich Moderatoren wie Geschlecht oder Kultur (westlich vs. asiatisch) nicht signifikant auswirkten $^{58}$.

\subsection{Meta-Analysen zur Fernseh- und Computerspielgewalt}

Bushman und Huesmann untersuchten 2006 mithilfe eines sehr ähnlichen metaanalytischen Vorgehens wie dem von Anderson und Bushman bei der Analyse von den Computerspielgewalteffekten den allgemeinen Einfluss von Gewaltmedieninhalten (Fernsehen, Filme, Computer- und Videospiele, Musik und Comics) auf verschiedene gewaltnahe Parameter (vgl. S 86 in dieser Arbeit). In 431 zwischen 1887 und 2000 veröffentlichten Studien mit zusammen N = 68.463 Teilnehmern fanden sie signifikante schwache bis mittlere positive Medien-

58 Im Rahmen dieser Meta-Analyse setzen sich die Autoren auch kritisch mit Publikationen von Ferguson und Kollegen auseinander (Ferguson, 2007a, 2007b; Ferguson \& Kilburn, 2009), die auf der Grundlage eigener Meta-Analysen keine signifikanten Effekte von Computerspielgewalt auf Gewaltverhalten erkennen wollen. Da der Kritik von Anderson et al. (2010) an den datenanalytischen Methoden von Ferguson und Kollegen beim Umgang mit gefundenen Studien vom Autor dieser Arbeit ausdrücklich zugestimmt wird, werden Fergusons Analysen und Interpretationen im Rahmen dieser Arbeit nicht näher dargestellt. 
effekte auf aggressives Verhalten ( $r=0.19)$, auf aggressive Gedanken $(r=0.18)$, aggressive Gefühle ( $r=0.27)$, körperliche Erregung (arousal) $(r=0.26)$, sowie schwache negative Effekte auf vermindertes prosoziales Verhalten (helpful behavior) $(r=-0.08)$. Diese Effekte fanden die Autoren sowohl bei Kindern (264 Studien mit $\mathrm{N}=50.312$ Teilnehmern) als auch bei Erwachsenen (167 Studien mit $\mathrm{N}=18.151$ Teilnehmern), wobei sich in einer varianzanalytischen Auswertung höhere Effektstärken der Gewaltmediennutzung bei Kindern zeigten, wenn die Studie Langzeiteffekte der Gewaltmediennutzung untersuchte (S. 351). Bei erwachsenen Studienteilnehmern zeigten sich dagegen stärkere kurzfristige Effekte. Bushman und Huesmann führen dies darauf zurück, dass kurzfristiges Priming durch Gewaltmedieninhalte besonders dann funktioniere, wenn ein bereits elaboriertes kognitives Netzwerk aggressiver Skripte, Überzeugungen und Schemata existiert. Dies sei bei Erwachsenen eher der Fall, während Kinder ein solches Netzwerk erst noch entwickeln müssen. Vor diesem Hintergrund interpretieren die Autoren die größeren Langzeiteffekte der Mediengewaltnutzung bei Kindern als erste Resultate der Etablierung eines solchen Netzwerkes (S. 350).

\subsection{Längsschnittstudien}

\subsection{Längsschnittstudien zum Fernsehen}

Neben den oben aufgeführten Meta-Analysen wurde in den letzten Jahren eine Reihe von Längsschnittstudien zur Gewaltmediennutzung von Kindern und Jugendlichen veröffentlicht, die die Langzeiteffekte der Mediengewaltnutzung auf das Verhalten in den Blick nehmen. So publizierten Johnson, Cohen, Smailes, Kasen und Brook (2002) eine Studie, in welcher N = 707 Probanden von 1975 bis 2000 vom sechsten bis zum dreißigsten Lebensjahr begleitet wurden. Dabei zeigte sich ein signifikanter Zusammenhang zwischen Fernsehkonsum $^{59}$ im Alter von 14 Jahren und Gewaltkriminalität ${ }^{60}$ im Alter von 22 Jahren bei Kontrolle wichtiger Faktoren (Vernachlässigung in der Kindheit, niedriges Familieneinkommen, geringe elterliche Bildung, Gefährlichkeit des

59 Dabei wurde im Rahmen von Interviews allein die Zeit erfragt, die die Probanden durchschnittlich vor dem Fernseher verbringen (Johnson, J. G. et al., 2002, S. 2469).

60 Gewaltkriminalität im Alter von 22 und 30 Jahren wurde durch Auswertung offizieller Strafregistereinträge der untersuchten Personen beim Federal Bureau of Investigation (FBI) erfasst (Johnson, J. G. et al., 2002, S. 2469). 
Wohnviertels und psychiatrische Auffälligkeiten der Jugendlichen, vorherige Auffälligkeit durch Gewaltverhalten und frühere Fernsehzeiten), sowie zwischen Fernsehkonsum im Alter von 22 Jahren und Gewaltkriminalität im Alter von 30 Jahren. Dieser Zusammenhang zeigte sich im Übrigen nicht zwischen Fernsehkonsum und reinen Eigentumsdelikten (Diebstahl, Vandalismus und Brandstiftung) (S. 2470). Obwohl Johnson et al. mit dieser Studie eindrucksvoll einen direkten Zusammenhang zwischen Fernsehnutzung und polizeilich registrierter Gewaltkriminalität belegen konnten, muss doch als Schwäche der Studie geltend gemacht werden, dass die Autoren lediglich die Fernsehzeit der Studienteilnehmer erhoben, nicht aber die Inhalte. Zwar belegen zahlreiche Studien einen eindeutigen Zusammenhang zwischen erhöhter Fernsehzeit und erhöhtem Gewaltmedienkonsum bei Kindern und Jugendlichen (vgl. etwa Gentile et al., 2004; Mößle et al., 2007), womit indirekt auf einen Effekt der Mediengewaltinhalte geschlossen werden kann. Jedoch bleiben Johnson et al. den direkten Beleg für diese Interpretation schuldig ${ }^{61}$. Zudem erscheint der Fokus der Studie auf den Fernsehkonsum im Alter mit 14 Jahren vor dem Hintergrund der aktuellen Erkenntnislage verfehlt. Zwar ist es umso bemerkenswerter, dass die Autoren auch beim Fernsehkonsum mit 14 noch Effekte fanden, eine parallele Auswertung der Effekte des kindlichen Fernsehkonsums wäre für die theoretische Debatte weitaus fruchtbarer gewesen, zumal solche Daten nach Angaben der Autoren offenbar vorlagen (2002, S. 2469 - 2470).

Eine differenziertere Erfassung der Art des Fernsehkonsums gelang Huesmann, Moise-Titus, Podolski und Eron (2003). Auf der Grundlage einer ursprünglich auf zwei Jahre angelegten Panelstudie zum Gewaltverhalten von $\mathrm{N}=557$ sechs- bis neunjährigen amerikanischen Grundschülerinnen und Grundschülern in den Jahren 1977 und 1978 (vgl. Huesmann, L. R. \& Eron, 1986; Huesmann, L. R., Lagerspetz \& Eron, 1984) wurde rund 15 Jahre später, zwischen 1992 und 1995, eine erneute Befragung aller noch auffindbaren nunmehr 21- bis 23-jährigen $\mathrm{N}=329$ Studienteilnehmer realisiert. Zur Messung der TV-Nutzung in der Kindheit nutzten die Autoren Selbstangaben der Kinder, die im Alter von acht Jahren in einer Liste von 80 aktuellen Fernsehsendungen ankreuzen konnten, wie häufig sie welche Sendung schauen und welche Sendung sie als ihre Lieblingssendung bezeichnen würden. Alle Sendungen wurden bezüglich ihres Gewaltgehaltes von Psychologie-Studenten beurteilt, so dass zur Fernsehnutzung der Kinder nicht nur bezüglich der genutzten Menge, sondern auch des Gewaltgehaltes Daten vorlagen (Huesmann, L. R. et al., 2003, S. 205). Zusätzlich wurde die Identifikation der Kinder mit Protagonisten bekannter ge- 
walthaltiger Fernsehsendungen gemessen sowie die Einschätzung der Kinder zum Realismus von Fernsehgewalt (S. 205 - 206). Die Messung der Fernsehnutzung der 15 Jahre später befragten jungen Erwachsenen geschah in ähnlicher Weise (vgl. S. 206). Aggressives Verhalten der Kinder wurde mithilfe eines getesteten Peer-Rating-Verfahrens erfasst, Gewaltverhalten der jungen Erwachsenen durch eine Kombination aus Selbst- und Fremdangaben sowie amtlicher Statistiken (Eintragungen ins staatliche Straf- beziehungsweise Verkehrsregister (S. 206 - 207).

Anhand von Regressions- und Cross-Lagged-Strukturgleichungsmodellen belegten Huesmann et al. signifikante Korrelationen zwischen Fernsehgewaltnutzung in der Kindheit und Aggressivität im Erwachsenenalter, selbst wenn die Intelligenz der Kinder und die Bildung der Eltern kontrolliert wurden. Dabei zeigte sich aggressives Verhalten im Erwachsenenalter besonders dann, wenn in der Kindheit eine Identifikation mit gewalttätigen TV-Protagonisten des gleichen Geschlechts vorlag und TV-Gewalt von den Kindern als eher realistisch eingeschätzt wurde. Diese Effekte galten für männliche wie weibliche Befragte gleichermaßen. Zwar fanden die Autoren im Rahmen ihrer Studie wie erwartet, dass frühe Gewaltauffälligkeit in der Kindheit sowohl Gewaltauffälligkeit im Erwachsenenalter wie auch Gewaltmediennutzung beeinflusst, doch konnten sie durch Verwendung von Cross-Lagged-Modellen zeigen, dass mit hoher Wahrscheinlichkeit ein kausaler Zusammenhang zwischen früher Gewaltfernsehnutzung und späterer Aggressivität besteht.

\subsection{Längsschnittstudien zur Computerspielnutzung}

Beleuchten die bisher diskutierten Längsschnittstudien den Zusammenhang zwischen (Gewalt-) Fernsehnutzung und Aggressivität, haben andere Autoren den Zusammenhang zwischen der Nutzung gewalthaltiger Computerspiele und Aggressivität im Längsschnitt untersucht. So publizierten Wallenius \& Punamäki (2008) eine Längsschnittstudie an N = 316 finnischen Kindern und Jugendlichen, die zweimal, zunächst im Alter von 10 Jahren (jüngere Alterskohorte) beziehungsweise 13 Jahren (ältere Alterskohorte), zwei Jahre später im Alter von 12 Jahren (jüngere Alterskohorte) und 15 Jahren (ältere Alterskohorte), befragt wurden. Mithilfe regressionsanalytischer Modelle ${ }^{62}$ untersuchten die Autoren

62 Nach dem Vorbild von Anderson und Anderson (1996) und Anderson und Dill (2000) verwendeten die Autoren ein regressionsanalytisches Verfahren zur ,Zerstörung“ eines Effektes (zum Beispiel von Mediengewalt auf Gewaltverhalten) durch schrittweises 
den Zusammenhang zwischen der Nutzung gewalthaltiger Computerspiele und gewalttätigem Verhalten gegenüber anderen (zur Operationalisierung und Validität der verwendeten Selbstauskunftsskalen siehe S. 288). Dabei fanden sie nach Kontrolle von Trait-Gewalt, und Alter der befragten Schülerinnen und Schüler longitudinale Effekte der Nutzung gewalthaltiger Computerspiele auf aggressives Verhalten, die aber durch das Geschlecht und die Eltern-KindKommunikation der Befragten unterschiedlich beeinflusst waren (S. 291). So zeigte sich bei Kontrolle weiterer wichtiger Einflussfaktoren bei Mädchen dann ein kausaler Zusammenhang zwischen Gewaltspielen und Gewaltverhalten, wenn sie von einer defizitären Kommunikation mit ihren Eltern berichteten. Jungen hingegen waren, entgegen der Vorannahmen der Autoren, dann besonders anfällig für Gewaltspieleffekte, wenn sie die Kommunikation mit ihren Eltern als gut beschrieben. Dahingegen zeigte häufige Gewaltspielnutzung bei Jungen mit schlechtem Kontakt zu den Eltern keine Effekte. Da Jungen dieser letzten Gruppe von vornherein ein hohe Aggressionsniveau aufwiesen, interpretieren Wallenius und Punamäki diesen Effekt dahingehend, dass Jungen dieser Gruppe aufgrund mangelnden elterlichen Engagements in der Medienerziehung bereits früher mit der Nutzung von Gewaltcomputerspielen angefangen haben (was sich im anfänglichen Aggressionsniveau zeige) und inzwischen gegenüber Spielgewalt desensibilisiert seien. Da Gewaltdesensibilisierung nicht direkt erhoben wurde, konnte diese Interpretation nicht im Rahmen der veröffentlichten Studie überprüft werden.

Ergebnisse einer dem Design der finnischen Studie von Wallenius und Punamäki ähnlichen Untersuchung publizierten Möller und Krahé im Jahr 2009. Dabei konnten die Autorinnen mithilfe einer Längsschnittstudie an N $=295$ Berliner Schülerinnen und Schüler siebter und achter Schulklassen (Durchschnittsalter zum ersten Messzeitpunkt: 13,3 Jahre) einen direkten längsschnittlichen Einfluss gewalthaltigen Computerspielens auf physische Aggressivität

Auspartialisieren anderer bekannter Einflussfaktoren. Beim Destructive Testing im Rahmen von Längsschnittanalysen mit 2 Messzeitpunkten wird nach Behauptung des Effektes eines zur Disposition stehenden zum Zeitpunkt t1 gemessenen Einflussfaktors gegenüber anderen Einflussfaktoren auf eine zu t2 gemessene abhängige Variable in einem letzten Schritt die zu tl gemessene abhängige Variable als Einflussfaktor in die Analyse aufgenommen. Da diese Variable bei relativ konstanten menschlichen Eigenschaften (wie zum Beispiel Gewaltverhalten) mutmaßlich den größten Einfluss auf die zu t2 gemessene abhängige Variable hat, ist die Chance mit diesem Verfahren besonders hoch, den zur Disposition stehenden Einflussfaktor zu ,zerstören“. Gelingt dies nicht, gilt der so geprüfte Einflussfaktor als robust. 
nachweisen ${ }^{63}$. Nach der Analyse des Computerspielverhaltens und Selbstangaben zum eigenen Gewaltverhalten der befragten Schülerinnen und Schüler zu zwei 30 Monate auseinander liegenden Messzeitpunkten (zum zweiten Messzeitpunkt nahmen noch $\mathrm{N}=143$ Schülerinnen und Schüler an der Befragung teil), kamen die Autorinnen zu dem Schluss, es könnte keine Bestätigung für die Hypothese gefunden werden, die Befunde der internationalen Forschung zur Korrelation zwischen Gewaltverhalten und Gewaltcomputerspielen sei darauf zurückzuführen, dass von Grund auf aggressivere Jugendliche aggressivere Spiele auswählten (Selektionshypothese) (S. 85). Vielmehr sehen sie in ihren Ergebnissen einen klaren Beleg für die „Sozialisations-Hypothese“, wonach diejenigen, die mehr Zeit mit Gewaltspielen verbringen, aggressiver werden (ebenda). Dabei zeigten sich signifikante Effekte der Nutzung von Gewaltcomputerspielen bereits bei relativ geringer Spielenutzung (ebenda). In ihrer Dissertation bemerkt Möller, die absoluten Effekte des Gewaltspielkonsums seien zwar als moderat zu bezeichnen, es müsse allerdings bedacht werden, dass es sich bei der Nutzung gewalthaltiger Videospiele „lediglich um einen Aspekt des allgemeiner gefassten Faktors Mediengewalt handelt"“ (2006, S. 7).

\subsection{Wirkungen von Filmen $u$ und Computerspielen im Längsschnitt}

In Längsschnittanalysen von Anderson, Gentile und Buckley (2007) sowie von Hopf, Huber und Weiß (Hopf, Huber \& Weiß, 2008) wurde versucht, die Effekte der Gewaltmediennutzung nicht entweder am Beispiel des Computerspielens oder des Film- beziehungsweise Fernsehkonsums zu analysieren, sondern beide Aspekte parallel zu untersuchen. Ein solches Vorgehen wird nicht nur der Lebensrealität von Kindern und Jugendlichen weitaus mehr gerecht, sondern es ermöglicht zudem, die Frage zu beantworten, bei welcher Art der Gewaltmediennutzung größere Effekte zu erwarten sind. Die Längsschnittstudie von Hopf, Huber und Weiß (2008) untersuchte den Zusammenhang der Nutzung von

63 Es handelt sich dabei um eine Nachfolgestudie der Dissertation von Möller (2006), die bereits nach einem Zeitraum von sechs Monaten Einflüsse des Computer- und Videospielens auf Aggressivität nachweisen konnte. Möller kam bereits in dieser Publikation zu dem Schluss, ihre Daten belegten ,eine Art „Abwärtsspirale“ der MedienkonsumAggressions-Beziehung (2006, S. 7). Aggressive Jugendliche zeigten, so Möller, zunächst ein verstärktes Interesse für gewalthaltige Medieninhalte, wobei die Nutzung dieser Inhalte wiederum zu einer Bekräftigung ihrer aggressiven Disposition führe (ebenda). Dabei betont Möller, dass bereits nach sechs Monaten der Effekt der Gewaltspielnutzung auf die Aggressivität größer war, als der Effekt der persönlichen Gewaltdisposition (Trait-Gewalt) auf die Auswahl von Gewaltspielen (ebenda). 
Gewaltmedien und aggressivem Verhalten von $\mathrm{N}=314$ im Durchschnitt 12jährigen (erster Messzeitpunkt Ende 1999/Anfang 2000) bis knapp 15-jährigen (zweiter Messzeitpunkt Ende 2002/Anfang 2003) Hauptschülerinnen und Hauptschüler mit gewaltdelinquentem Verhalten. Die Beschränkung auf Schülerinnen und Schüler dieser Schulform bietet den Vorteil, eine durch Gewaltprävalenz besonders belastete Gruppe zu untersuchen (vgl. etwa Baier et al., 2006; wie auch die Studienübersicht bei Hopf et al., 2008, S. 82). Im Rahmen der Studie zeigte sich, dass die Nutzung gewalthaltiger Fernsehsendungen, von Gewalt- beziehungsweise Horrorfilmen und gewalthaltigen Computerspiele einen direkten längsschnittlichen Effekt sowohl auf Gewaltverhalten der Befragten in der Schule (Schulgewalt) als auch auf (selbst berichtete) Gewaltdelinquenz hatte (S. 90). Eine separate Analyse der Effekte einzelner Medien ergab, dass Gewaltcomputerspielnutzung den stärksten Effekt auf selbstberichtete Gewaltdelinquenz hatte (S. 87), während die Nutzung von Gewalt- beziehungsweise Horrorfilmen am stärksten mit Schulgewalt verknüpft war (S. 86). Für die Nutzung von Fernsehgewalt ergaben sich die schwächsten, teilweise nur indirekte Effekte (S. 86 - 87). Damit liefert die Studie von Hopf et al. nicht nur einen weiteren Beleg für einen kausalen Zusammenhang zwischen Gewaltmediennutzung und Gewaltverhalten, sondern gibt Hinweise auf unterschiedlich große Effekte unterschiedlicher Medien. Leider unterlassen es die Autoren, separate Medieneffekte auf Gewaltverhalten gleichzeitig zu modellieren. Damit hätten die Ergebnisse von Mößle et al. (2007, S. 111 - 112) auch längsschnittlich überprüft werden können, wonach die Nutzung von Gewaltcomputerspielen stärker kausal mit Gewaltdelinquenz verbunden ist, während die Nutzung von Horror- und Gewaltfilmen zumindest bei Jugendlichen eher als Indikator für hohe Gewaltakzeptanz und einen mit Gewaltprävalenz verknüpften Lebensstil zu sein scheint.

Unterstützung für diese Interpretation liefern Daten der amerikanischen Längsschnittstudie von Anderson, Gentile und Buckley (2007). Diese zu zwei Messpunkten im Abstand von durchschnittlich fünf Monaten durchgeführte Studie an $\mathrm{N}=430$ Dritt-, Viert- und Fünftklässlern (Durchschnittsalter zum ersten Messzeitpunkt: 9,6 Jahre) zeigte direkte längsschnittliche Effekte der Nutzung von Gewaltcomputerspielen und Gewaltfilmen auf aggressive Attributionen der Befragten, verbales und physisches Gewaltverhalten ${ }^{64}$. Mithilfe von regressionsanalytischen Verfahren zeigten die Autoren, dass in direkter

64 Verbale Gewalt wurde dabei mithilfe eines peer-nomination-Verfahrens durch die Mitschüler gemessen, physische Gewalt mithilfe eines Indexes aus peer-nomination, Lehrer/innen- und Selbstangaben (Anderson, C. A. et al., 2007, S. 97 - 101). 
Konkurrenz der Medieneffekte untereinander (Computerspiele vs. Film/Fernsehen) gewalthaltige Computerspiele einen signifikanten Einfluss behalten, während der direkte Effekt der Filmnutzung nicht mehr signifikant wurde (S. 117). Anderson et al. warnen allerdings davor, dies als Beleg eines Nulleffektes der Gewaltfilmnutzung zu werten. Vielmehr weise das Ergebnis einfach auf den größeren Effekt der Computerspielnutzung hin, der wegen der hohen Korrelation von Gewaltcomputerspiel- und Gewaltfilm-/-fernsehnutzung den Effekt der Gewaltfilm- oder -fernsehnutzung unterdrücke (ebenda). Neben der Dissertation von Möller (2006) ist die Publikation von Anderson et al. die zweite, die bereits Gewaltmedieneffekte nach wenigen Monaten nachweisen konnte. Bezog sich die Studie von Möller auf jugendliche Schülerinnen und Schüler, befanden sich die Studienteilnehmerinnen und -teilnehmer von Anderson et al. (zumindest zu einem sehr großen Teil) noch im Kindesalter, so dass nach den Ergebnissen dieser beiden Studien davon ausgegangen werden muss, dass Gewaltmedieneffekte im Alter von neun bis 16 Jahren bereits nach einem recht kurzen Zeitfenster mit regelmäßiger Gewaltmediennutzung auftreten können.

\subsubsection{Zusammenfassung zur Wirkung von Mediengewalt auf Aggressivität}

Wie gezeigt werden konnte, ist die Wirkung sowohl rein rezeptiver als auch interaktiver Mediengewalt sowohl meta-analytisch als auch längsschnittlich auf aggressives Verhalten von Erwachsenen als auch insbesondere von Kindern eindeutig belegt worden. Wiesen erste meta-analytische Studien darauf hin, dass der Effekt von Fernseh- oder Filmgewalt auf Gewaltverhalten oder gewaltnahe Parameter etwas größer ist, als der Effekt von interaktiver Gewalt, weisen aktuelle Studien darauf hin, dass Computer- und Videospielgewalt in aktuellen Spielen Gewaltverhalten stärker beeinflusst als rein rezeptive Gewalt. Zwar stellt, so Kunzcik und Zipfel in ihrem Review zur Gewaltwirkungsforschung bis 2004, ,[...] Mediengewalt nur einen Faktor innerhalb eines komplexen Bündels von Ursachen für die Entstehung gewalttätigen Verhaltens dar“ (S. 289). Dabei ist allerdings zu berücksichtigen, „dass sich hinter einem geringen Einfluss für den Durchschnitt der Medieninhalte und Rezipienten durchaus stärkere Effekte für bestimmte Inhalte und bestimmte Rezipienten verbergen können [...]“ (ebenda). Und die Autoren folgern:

„Auswirkungen von Mediengewalt auf Aggressionsverhalten sind am ehesten bei jüngeren, männlichen Vielsehern $\mathrm{zu}$ erwarten, die in Familien mit hohem Fernseh(-gewalt-)konsum aufwachsen und in ihrem unmittelbaren sozialen Umfeld (d. h. 
in Familie, Schule und Peergroup) viel Gewalt erleben (so dass sie hierin einen „,normalen“ Problemlösungsmechanismus sehen), bereits eine violente Persönlichkeit besitzen und Medieninhalte konsumieren, in denen Gewalt auf realistische Weise und/oder in humorvollem Kontext gezeigt wird, gerechtfertigt erscheint und von attraktiven, dem Rezipienten möglicherweise ähnlichen Protagonisten mit hohem Identifikationspotenzial ausgeht, die erfolgreich sind und für ihr Handeln belohnt oder zumindest nicht bestraft werden und dem Opfer keinen sichtbaren Schaden zufügen (,saubere Gewalt)“ (Kunczik, M. \& Zipfel, 2004, S. 289).

\subsubsection{Prosoziale Effekte}

\subsubsection{Theoretischer Hintergrund}

Vor dem Hintergrund der sozial-kognitiven Lerntheorie und damit verknüpfter theoretischer Ansätze erscheint es zunächst trivial, dass Mediennutzung nicht nur geeignet ist, problematische Lernprozesse anzuregen und zu unterstützen, die antisoziales und aggressives Verhalten der Probanden zur Folge haben, sondern dass Mediennutzung ebenso das Potential zur Initialisierung und Vertiefung prosozialer Lernprozesse innewohnt. So konnte bereits gezeigt werden, dass im Rahmen der schulischen Bildung insbesondere interaktive Medien erfolgreich eingesetzt wurden, um Lernen anzuregen und $\mathrm{zu}$ Lerninhalte $\mathrm{zu}$ festigen (vgl. S. 75 in dieser Arbeit). Ebenso sind aus dem Bereich der Gesundheitsfürsorge und der Therapie verschiedener Krankheiten Wirkungen des Einsatzes klassischer und interaktiver Medien belegt (vgl. statt vieler etwa Bandura, 1998, 2004). Abseits des schulischen Lernens und der Gesundheitsfürsorge wird immer wieder auch die Rolle der Medien als Vermittlungsinstanz prosozialer Inhalte und Verhaltensmodelle betont. Wird etwa in der Schule oder in der Therapie beispielsweise ein Computerprogramm sehr zielgerichtet eingesetzt, um effektiv Vokabeln zu lernen oder einem an Diabetes erkrankten Kind ein adäquates Ernährungs- und Selbstmedikationsverhalten beizubringen, sind ebenso nichtintendierte prosoziale Wirkungen von Mediennutzung denkbar, die sich aus der Nutzung von prosozialen Medieninhalten ergeben. Analog zum General Aggression Model von Anderson und Bushman erarbeiteten Gentile et al. (2009) (ebenfalls unter Mitwirkung von Bushman und Anderson) das General Learning Model, mit dessen Hilfe die Akquisition prosozialer Skripte, Einstellungen und Verhaltensweisen theoretisch beschrieben wird. 


\subsection{Fernsehen}

Die prosozialen Effekte der Fernsehnutzung sind empirisch relativ gut erforscht, wenn auch nicht annähernd so ausführlich wie die antisozialen Effekte (Mares \& Woodard, 2007, S. 297). Mares und Woodard (2001, 2007) untersuchten im Rahmen einer Aktualisierung der ersten großen Meta-Analyse von Hearold (1986) die Studienlage zur Wirkung prosozialer Fernsehinhalte auf prosoziales Verhalten von Kindern. Dabei fanden sie 34 unabhängige experimentelle beziehungsweise Befragungsstudien mit insgesamt $\mathrm{N}=5.473$ Studienteilnehmerinnen und -teilnehmern, in denen als abhängige Variable prosoziales Verhalten gemessen wurde. Als prosozial sowohl bezüglich der gezeigten Inhalte als auch bezüglich der gemessenen abhängigen Variablen definierten ${ }^{65}$ die Autorinnen dabei erstens ,positive Interaktionen (positive interaction)“, zum Beispiel freundschaftliches miteinander Spielen oder auch friedliches Konfliktlösungsverhalten (Mares \& Woodard, 2001, S. 184). Zweitens definierten die Autoren altruistisches Verhalten (aufopferungsvolle Freundlichkeit, Teilen, Schenken, Helfen inkl. Anbieten von Hilfe sowie Trösten) als prosozial. Drittens wurde die Reduzierung von gesellschaftlichen Stereotypen sowohl auf Medieninhaltsseite wie auch auf Effektseite ${ }^{66}$ als prosozial definiert. Und schließlich nahmen die Autorinnen auch Studien zur Wirkung prosozialer Medieninhalte auf Aggressivität in die Analyse auf, da vermutet werden konnte, dass aggressives Verhalten (verbale und physische Aggressivität) durch prosoziale Medieninhalte gemindert wird ${ }^{67}$.

Insgesamt fanden Mares und Woodard signifikante moderate Effekte prosozialer Inhalte auf das Verhalten von Kindern $(r=0.27)$. Die größten Effekte berichten sie für Mediendarstellungen altruistischen Verhaltens auf altruistisches Verhalten der kindlichen Rezipienten. Dies sei, so die Autorinnen, deshalb wenig überraschend, als bei dieser Unterdimension prosozialen Verhaltens am ehesten eine inhaltliche Übereinstimmung zwischen Treatment (Medieninhalt) und

65 Die genauen Definitionen von Prosozialität unterscheiden sich in den beiden verfügbaren Veröffentlichungen der Autoren leicht. Insofern wurde darauf geachtet, alle in beiden Veröffentlichungen genannten Unterdimensionen zu erwähnen.

66 Auf Effektseite wurden hier Messungen zu Einstellungen oder Verhaltensweisen gegenüber häufig als stereotyp dargestellten Personen, Gesellschaftgruppen oder Gesellschaften einbezogen.

67 Mit der Fokussierung auf diese drei Bereiche prosozialen Verhaltens unterscheiden sich die Autoren recht deutlich von der Vorläufer-Studie von Hearold (1986). 
Effektvariable gegeben sei (2001, S. 184). Diese Interpretation erscheint auch vor dem Hintergrund der sozial-kognitiven Lerntheorie Banduras plausibel, wird hier doch postuliert, dass Modell-Lernen dort am besten funktioniert, wo eine Übertragung eines medial präsentierten Modells besonders einfach ist. Darüber hinaus fanden Mares und Woodard auch signifikante schwache Effekte prosozialer Fernsehprogramme auf positive Interaktionen, Reduzierung von Stereotypen und Reduzierung von Gewalt. Mit Blick auf das Alter der jeweiligen Studienteilnehmer kamen sie zu dem Schluss, dass die Wirkung prosozialer Medienbotschaften offenbar im Alter von drei Jahren recht gering ist und bis zum Alter von sieben Jahren deutlich zunimmt. Bei zwölfjährigen Kindern seien bereits wieder kleinere Effekte zu beobachten, die bei Jugendlichen dann noch einmal etwas kleiner würden (2007, S. 297). Dies sei, so die Autorinnen, ein deutlich anderer Befund als die von Paik und Comstock (1994) veröffentlichten Daten, nach denen Gewalteffekte der Gewaltmediennutzung bereits sehr früh zu beobachten seien und kleine Kinder als besonders anfällig für Gewalteffekte seien (Mares \& Woodard, 2007, S. 296). Mares und Woodard führen diesen Unterschied darauf zurück, dass erst mit Erreichen eines bestimmten Alters Kinder in der Lage seien, den Gehalt prosozialer Medienbotschaften so zu erfassen, so dass sie in ihrem persönlichen Umfeld ein entsprechendes Verhalten entwickeln könnten (Mares \& Woodard, 2007, S. 297).

Im Vergleich der Effekte prosozialer Inhalte mit aggressiv-antisozialen Inhalten zeigen sich nach Einschätzung der Autoren keine deutlichen Unterschiede. Ermittelte Hearold (1986) in ihrer Meta-Analyse noch fast doppelt so starke Effekte prosozialer Inhalte gegenüber antisozialen Inhalten und argumentierte damit im Sinne der Hypothese starker prosozialer Effekte des Fernsehens, schließen Mares und Woodard, in der Größe der Effekte sei ihre Studie in etwa mit der Studie von Paik und Comstock vergleichbar, während die Effektstärke (=Power) von Mediengewalt auf Gewaltverhalten womöglich noch etwas höher einzuschätzen sei als die Effektstärke prosozialer Inhalte auf prosoziales Verhalten (Mares \& Woodard, 2001, S. 185). Insgesamt, so die Autorinnen, müsse aber berücksichtigt werden, dass die gleiche Effektstärke pround antisozialer Inhalte noch nichts über das inhaltliche Angebot im Fernsehen und dessen Nutzung aussage (Mares \& Woodard, 2007, S. 296). Unter Verweis auf US-amerikanische Inhaltsanalysen des Fernsehprogramm sowie Studien zur Fernsehnutzung von Kindern geben sie zu bedenken, dass angesichts eines hohen Levels häufig humoristisch oder glorifiziert dargestellter Gewalt mit entsprechend hoher Nutzung durch Kinder und angesichts eines recht überschaubaren Angebotes explizit prosozialer Inhalte in Frage stünde, inwieweit sich die Effekte prosozialer Inhalte in der Lebenswirklichkeit von Kindern tatsächlich 
niederschlagen (S. 281 - 283). Zumal Folgendes festzustellen sei: Wenn prosoziale Inhalte im Fernsehen gezeigt werden, dann häufig im Kontext von Gewaltdarstellungen, wenn Gewalt gezeigt wird, dann nur selten im Kontext gewaltkritischer Botschaften (S. 282).

\subsection{Computerspiele}

Bezüglich der Effekte prosozialer Inhalte und Konzepte von Computerspielen besteht nach Meinung von Hogan und Strasburger (2008, S. 547) wie auch Gentile et al. (2009, S. 755) ein großes Defizit in der Forschung. Zwar erscheint es berechtigt, wenn Walsh und Gentile bezüglich der Effekte des Computerspielens folgern:

"[...] video games could have large effects, some of which are intended by game designers, and some of which may not be intended. [...] Given the fact that video games are able to have several positive effects, it should come as no surprise that they also can have negative effects" (Walsh \& Gentile, 2004).

Belastbare empirische Erkenntnisse zu prosozialen Wirkungen von Computerspielen finden sich jedoch kaum. Theoretisch, so Gentile et al. (S. 754), bieten Computerspiele aufgrund ihrer Beschaffenheit als interaktive Medien, eine ganze Reihe von Möglichkeiten, mit und aus ihnen zu lernen. Mithilfe eines internationalen Forschungsprogramms aus einer Querschnittstudie, einer Längsschnittstudie und einem Experiment, haben die Autoren versucht, prosoziale Effekte des Computerspielens nachzuweisen. So zeigten sie anhand von Regressionsdaten einer Befragung von $\mathrm{N}=727$ durchschnittlich 13-jährigen Schülerinnen und Schülern aus Singapur, dass das Spielen prosozialer Computerspiele $^{68}$ positiv mit allen in der Studie verwendeten Indikatoren für prosoziales Verhalten korreliert war (Hilfsverhalten, Kooperation und Teilen, Empathie, emotionale Aufmerksamkeit). Ein negativer Zusammenhang zeigte sich dagegen zwischen der Nutzung prosozialer Computerspiele und Indikatoren für Aggressivität und aggressives Verhalten (S. 756), wie auch zwischen der Nutzung gewalthaltiger Computerspiele und den prosozialen Indikatoren Hilfsverhalten und Empathie ${ }^{69}$ (zwischen Gewaltspielnutzung und Emotionaler Auf-

68 Der prosoziale Charakter der Spiele wurde dabei durch ein mit Expertenratings abgesichertes Selbstratingverfahren der befragten Schülerinnern und Schüler festgestellt (Gentile et al., 2009, S. 756).

69 Einen Befund diskutieren die Autoren im Rahmen Ihres Artikels leider nicht. So zeigte sich ein signifikanter, negativer Zusammenhang zwischen der wöchentlichen Spielzeit und allen Indikatoren von Prosozialität. Da die Nutzung sowohl gewalthaltiger wie auch 
merksamkeit sowie Kooperation und Teilen zeigten sich keine signifikanten $\mathrm{Zu}$ sammenhänge) (ebenda).

Um die Querschnittsbefunde auch kausal interpretieren zu können, führten Gentile et al. zusätzlich eine Längsschnittuntersuchung an $N=780$ Fünftklässlerinnen und Fünftklässlern sowie $\mathrm{N}=1.050$ Acht- sowie Elftklässlerinnen und klässlern durch. Der erste und der zweite Messzeitpunkt lagen drei bis vier Monate auseinander. Dabei fanden Gentile et al. auch einen längsschnittlichen Zusammenhang zwischen der Nutzung prosozialer Computerspiele und Prosozialität (S. 757), wobei die Autoren von einer fortwährenden Interaktion der Nutzung prosozialer Inhalte und Prosozialität in Form einer ständigen Verstärkung ausgehen ${ }^{70}$. In einer dritten Teilstudie testeten die Autoren schließlich, wie weit, analog zu Befunden des General-Aggression-Models, empirische Umsetzungen des General-Learning-Ansatzes neben Langzeiteffekten auch Kurzzeiteffekte finden können. Zu diesem Zweck wurde ein Experiment mit $\mathrm{N}=161$ durchschnittlich 19-jährigen Studentinnen und Studenten durchgeführt, in dem die kurzfristigen Auswirkungen des Spielens prosozialer, gewalthaltiger und neutraler Spiele geprüft wurden. Es zeigte sich, dass das 20-minütige Spielen eines im Rahmen einer Pilotstudie als prosozial eingestuften Spiels zu größerer Hilfsbereitschaft und weniger Missgunst führte, während Spielerinnen und Spieler eines Gewaltspiels signifikant weniger hilfsbereit und signifikant missgünstiger waren als Spielerinnen und Spieler eines prosozialen Spiels (S. 759 $760)^{71}$. Insgesamt sehen die Autoren das von ihnen postulierte General-Learning-

prosozialer Spiele mit gesonderten Koeffizienten ebenfalls in die Regression mit eingegangen war, zeigt sich hier offenbar ein vom Spielinhalt unabhängiger negativer $\mathrm{Zu}$ sammenhang zwischen reiner Spielzeit und Prosozialität. Dieser Befund, der - zumindest bezüglich der Empathie - auch im Rahmen anderer Studien offenbar wurde (Kleimann \& Mößle, 2008), ist an anderer Stelle dahingehend interpretiert worden, dass wenig empathische Kinder und Jugendliche sich aufgrund dieses Defizits stärker in virtuelle Welten flüchten als ihre Altersgenossen, wodurch Empathiemangel und weitere soziale Kompetenzdefizite sich weiter verstärken (Mößle \& Kleimann, 2009, S. 40 - 41).

70 Insgesamt erscheint dieser Studienteil allerdings wenig elaboriert. So wird weder dem Umstand der recht großen Altersspanne der teilnehmenden Schüler/innen theoretisch oder datenanalytisch überzeugend Rechnung getragen, noch werden die datenanalytischen Befunde hinreichend deskriptivstatistisch eingeführt oder inferenzstatistisch abgesichert. So hätte beispielsweise ein Cross-Lagged-Modell weitere Auskunft über den genauen Interaktionsmechanismus zwischen Prosozialität und der Nutzung prosozialer Computerspiele geben können.

71 Gestestet wurde dies mithilfe eines Tests, in dem die Probanden im Anschluss an das 20minütige Spieles eines Computerspiels eine Reihe von Puzzle-Aufgaben für einen angeblichen Mitspieler auswählen sollten. Den Probanden wurde erklärt, der Mitspieler würde 10 Dollar gewinnen, wenn er die Aufgaben richtig löst. Die Probanden sollten aus einem Set von 10 leichten, 10 mittelschweren und 10 schweren Puzzle-Aufgaben 11 Aufgaben 
Model durch die drei Teilstudien vorläufig bestätigt und kommen somit zu dem Schluss, dass die Frage positiver oder negativer Wirkungen des Computerspieles durch die Art der genutzten Spiele bestimmt wird (S. 761). Zugleich weisen sie darauf hin, dass unabhängig vom Inhalt der Spiele lange Spielzeiten zu negativen Displacement-Effekten führen können, indem beispielsweise schulische Leistung gemindert oder die Entwicklung sozialer Kompetenzen reduziert werden kann (ebenda).

\subsubsection{Zusammenfassung zu Medienwirkungen auf Sozialverhalten}

Insgesamt lässt sich festhalten, dass die Nutzung rezeptiver und interaktiver Medien signifikante Effekte auf das Sozialverhalten von Menschen hat, was im vorangegangenen Abschnitt besonders am Beispiel von Kindern und Jugendlichen gezeigt wurde. Dabei zeigt sich, das insbesondere lerntheoretische Ansätze im Sinne von Banduras sozial-kognitiver Lerntheorie geeignet sind, Medieneffekte auf das Sozialverhalten zu erklären, insbesondere wenn sie, wie das General-Aggression-Model von Anderson und Bushman, weitere wichtige Theorien zur affektiven Medienwirkung (beispielsweise Zillmans ExcitationTransfer-Theorie), zur Entwicklung kognitiver Strukturen (Cognitive Neoassociation Theory; Skripttheorie) und zur Entstehung sozialer Interaktionsprozesse (Social Interaction Theory) berücksichtigen. Obwohl theoretisch und empirisch sowohl prosoziale als auch antisoziale Effekte begründet und gefunden werden können, muss dabei berücksichtigt werden, dass die Nutzung explizit antisozial-gewalthaltiger Medien unter Kindern und Jugendlichen zumindest im Fernsehen die Nutzung explizit prosozialer Medien deutlich überwiegt. Bezüglich der Computerspielnutzung gibt es zwar deutliche Hinweise auf eine breite Nutzung gewalthaltiger Spiele (vgl. S. 36 und 43 in dieser Arbeit), jedoch fehlen bisher Studien, in denen die Nutzung explizit prosozialer Spiele repräsentativ erhoben wurde. Insgesamt spricht jedoch vieles dafür, dass prosoziale Medieneffekte, obschon lerntheoretisch genauso gut begründbar wie anti-

für den Mitspieler heraussuchen. Der durchschnittliche Schwierigkeitsgrad eines vom Probanden ausgewählten Aufgabensets galt als Indikator für Hilfsbereitschaft (Helpful = viele leichte Aufgaben) bzw. Missgunst (Hurtful = viele schwere Aufgaben). „Hurtful“ wurde hier als „Missgunst“ bzw. „missgünstig“ ins Deutsche übertragen, da das Auswählen eines schweren Puzzles aus Sicht des Autors dieser Arbeit weniger ein Anzeichen von Schädigungsabsicht des Probanden ist, als vielmehr ein Anzeichen dafür, dass dem vorgeblichen Mitspieler ein leichtes Gewinnen der 10 Dollar nicht gegönnt wurde. 
soziale Effekte, im Lebensalltag von Kindern und Jugendlichen eine kleinere Rolle spielen als antisoziale Effekte.

\subsection{Medienwirkungen auf physische Parameter}

Jenseits der kommunikationswissenschaftlichen Diskussion über Mediennutzungseffekte auf Kinder und Jugendliche existiert seit einigen Jahren eine weitgehend eigenständige Forschungstradition zu Medieneffekten auf Kindern und Jugendliche im Bereich der Pädiatrie und Jugendmedizin sowie der Psychiatrie. Hier wird vor allem der Zusammenhang zwischen Mediennutzung und der körperlich-seelischen Befindlichkeit und Entwicklung von Kindern und Jugendlichen diskutiert. Unbestritten ist, dass insbesondere die tägliche Beschäftigung mit $\mathrm{AV}$ - und IT-Medien $\mathrm{zu}$ verschiedenen körperlichen Beschwerden führen kann. Diese aus der Arbeitswelt bekannten Phänomene zeigen sich auch für Fernseh- und Computernutzung von Kindern und Jugendlichen (ein Überblick bei Egmond-Fröhlich et al., 2007). So wurde inzwischen die Korrelation zwischen kindlicher (Tazawa \& Okada, 2001) und jugendlicher (Hakala, Rimpela, Saarni \& Salminen, 2006; Sjolie, 2004) Computerspielnutzung mit diversen muskuloskeletalen Beschwerden belegt. Aber auch häufiger Fernsehkonsum von Schulkindern ist mit Beschwerden aus diesem Bereich, insbesondere mit Rückenschmerzen korreliert (Kristjansdottir \& Rhee, 2007; Mohseni-Bandpei, Bagheri-Nesami \& Shayesteh-Azar, 2007; Sjolie, 2004; Troussier, Davoine, de Gaudemaris, Fauconnier \& Phelip, 1994). Zudem weisen medizinische Studien bei Schulkindern auf deutliche Anzeichen von Augenermüdung und Augenbeschwerden nach längerer Computernutzung hin (Misawa, Shigeta \& Nojima, 1991) und berichten von signifikant häufigeren Kopfschmerzen bei Schulkindern mit häufiger Fernsehnutzung (Toyran, Ozmert \& Yurdakök, 2002). Darüber hinaus liegt inzwischen eine Reihe von Studien vor, die einen kausalen Zusammenhang zwischen der Nutzung elektronischer Medien und der Übergewichtigkeit sowie einem gestörten Essverhalten von Kindern und Jugendlichen nahelegen, zwischen Mediennutzung und Bewegungsarmut, zwischen Mediennutzung und Schlafstörungen sowie zwischen Mediennutzung und der allgemeinen emotionalen Belastung von Kindern und Jugendlichen. 


\subsubsection{Schlafstörungen}

Einschlafprobleme und Schlafstörungen gelten in der pädiatrischen Literatur als Auslöser oder Verstärker verschiedener psychischer, sozialer oder medizinischer Probleme insbesondere von Kindern im Grundschulalter (vgl. statt vieler Lehmkuhl, Wiater, Mitschke \& Fricke-Oerkermann, 2008; Stein, Mendelsohn, Obermeyer, Amromin \& Benca, 2001). So gilt aus Schlafstörungen resultierende Tagesmüdigkeit allgemein als signifikanter Prädiktor für defizitäre motorische und kognitive Leistungen (Pilcher \& Huffcutt, 1996) ${ }^{72}$ und - bezogen insbesondere auf Grundschulkinder - als Prädiktor für Aufmerksamkeits- und Schulleistungsprobleme (Owens, Spirito, McGuinn \& Nobile, 2000; Stein et al., 2001) sowie als Prädiktor verschiedener Verhaltensprobleme, zum Beispiel Aggressivität (Chervin, Hedgera, Dillonb \& Pituch, 2000; Stein et al., 2001). Dabei wurde wiederholt ein Zusammenhang zwischen Mediennutzung und (Ein-)Schlafproblemen von Kindern und Jugendlichen gefunden (Johnson, Jeffrey G., Cohen, Kasen, First \& Brook, 2004; Owens et al., 1999).

Mehrere Studien konnten zeigen, dass besonders extensive Fernsehnutzung durch Kinder zu späteren Einschlafzeiten und daraus resultierend kürzeren Schlafzeiten führt (Adam, Snell \& Pendry, 2007; Crönlein et al., 2007; Gaina et al., 2007; Heins et al., 2007; Li et al., 2007) sowie zu einer Minderung der Schlafeffizienz (Dworak, Schierl, Bruns \& Struder, 2007). Crönlein et al. (2007) wiesen dabei darauf hin, dass neben den reinen Nutzungszeiten auch die jeweils genutzten Inhalte zu beachten sind, da in ihrer Studie an $\mathrm{N}=137$ Jugendlichen Schlafzeiten wie auch verschiedene Einschlafprobleme, subjektives Empfinden der Schlafqualität und subjektives Empfinden der eigenen Leistungsfähigkeit mit den konkret genutzten TV-Medieninhalten in unterschiedlicher Form korreliert

72 Pilcher und Huffcutt (1996) untersuchten den Einfluss von Schlafdeprivation auf Stimmung, kognitive und motorische Leistungen im Rahmen einer Meta-Analyse von $\mathrm{N}=19$ zwischen 1986 und 1993 durchgeführten Schlafdeprivationsexperimenten an $\mathrm{N}=1.932$ erwachsenen Probanden. Dabei zeigten sie, dass sowohl kurzfristige wie auch langfristige Schlafdeprivation signifikant negative Effekte auf die Stimmung der Probanden wie auch deren kognitive und motorische Fähigkeiten hat (S. 323), wobei sich die der größte Effekt bei der Stimmung der Probanden zeigte, der zweitgrößte Effekt bei kognitiven Leistungen und der schwächste Effekt bei motorischen Leistungen. Alle gefundenen Effekte werden von den Autoren als „,nicht marginal“ bezeichnet. Williamson und Feyer (2000) verglichen die Effekte zwischen Schlafdeprivation und Alkoholkonsum und kamen zu dem Schluss, dass kognitive und motorische Leistungen von erwachsenen Probanden nach kurzem Schlafentzug ungefähr denen von Probanden mit einer Blutalkoholkonzentration von 0,5 Promille entsprachen, während längerer Schlafentzug zu Leistungen führte, die mit Probanden vergleichbar waren, die eine Blutalkoholkonzentration von 1,0 Promille aufwiesen. 
waren. Auch intensive Computerspielnutzung ist mit kürzeren Schlafzeiten von Kindern (Heins et al., 2007) (33 $^{73}$ und kürzeren Schlafzeiten von Jugendlichen (Rehbein, Kleimann \& Mößle, 2009a) korreliert. Zudem konnten Dworak et al. (2007) experimentell nachweisen, dass längeres Computerspielen für geringere Schlafqualität und vermittels dieses Faktors für schlechtere verbale Erinnerungsleistungen von Jugendlichen (Durchschnittsalter: 13,5 Jahre) verantwortlich gemacht werden kann. Einen deutlichen Mediennutzungseffekt auf Schlafzeiten, Schlafqualität und Schlafstörungen fanden verschiedene Studien besonders dann, wenn Kinder über eigene elektronische Mediengeräte im Zimmer berichteten (Lehmkuhl et al., 2008; Li et al., 2007; Owens et al., 1999).

\subsection{2 Übergewicht}

Ein Zusammenhang zwischen Mediennutzung, Übergewichtigkeit und Bewegungsarmut kann inzwischen als belegt gelten, wobei die Stärke des $\mathrm{Zu}$ sammenhangs als schwach $\mathrm{zu}$ bezeichnen ist. Als Ursache für diesen $\mathrm{Zu}$ sammenhang gelten insbesondere der durch die Mediennutzung verursachte Bewegungsmangel (Burke et al., 2006; Epstein et al., 2008; Rey-López, VicenteRodríguez, Biosca \& Moreno, 2008) sowie eine durch Fernsehwerbung verstärkte falsche Ernährung (vgl. statt vieler Hastings, Gerard, McDermott, Angus, Stead \& Thomson, 2007). Die pädiatrische Literatur der letzten Jahre beklagt einen eindeutigen Trend zu mehr Übergewichtigkeit (Ambler, 2004; Bundred, Kitchiner \& Biuchan, 2001; The Henry J. Kaiser Foundation, 2004; World Health Organisation, 2000) und Bewegungsmangel (ein Überblick der internationalen Studienlage bei Bös, 2003) unter Kindern und Jugendlichen verschiedener Industriestaaten und sieht in der Nutzung elektronischer Medien einen wichtigen Einflussfaktor auf beide Entwicklungen.

Tatsächlich kamen Marshall, Biddle, Gorely, Cameron und Murdey (2004) in einer Meta-Analyse von 107 Querschnitts-, Längsschnitts- und Interventionsstudien zum Zusammenhang der Fernseh-, Computer- und Videospielnutzung mit Körperfettgehalt ${ }^{74}$ und Bewegungsarmut von Kindern und Jugendlichen zum

73 Erwähnt sei in diesem Zusammenhang auch die Studie von Tazawa und Okada (2001), in der 1.143 japanische Schulkinder zwischen sechs und elf Jahren u. a. auf körperliche Indikatoren für Schlafmangel (zum Beispiel deutliche dunkle Augenringe) kinderärztlich untersucht wurden. Computerspielen war demnach signifikant mit dem Vorhandensein von Schlafmangelindikatoren korreliert.

74 Der Körperfettgehalt gilt als zuverlässiger Indikator gesundheitsrelevanter Übergewichtigkeit. Er wird im Rahmen größerer epidemiologischer Studien oder 
dem Schluss, dass sowohl zwischen Fernsehnutzung, Körperfettgehalt und Bewegungsarmut als auch zwischen Computerspielnutzung, Körperfettgehalt und Bewegungsarmut ein signifikanter Zusammenhang besteht. Die mittlere Effektstärke zwischen Fernsehnutzung und Körperfettgehalt geben die Autoren mit $r_{\mathrm{c}}^{75}=0.08$ an, die mittlere Effektstärke zwischen Computerspielnutzung und Körperfettgehalt mit $r_{\mathrm{c}}=0.13$ (S. 1241). Fernsehnutzung war nach dieser Studie mit $r_{\mathrm{c}}=-0.13$ mit physischer Aktivität korreliert, Computerspielnutzung mit $r_{\mathrm{c}}=-0.14$ (S. 1242). Der von Marshall et al. gefundene schwache, obschon signifikante Zusammenhang zwischen Mediennutzung und Körperfettgehalt, der von anderen Autoren als Indikator für eine Verstärkungswirkung der Mediennutzung ohnehin genetisch vorhandener Prädisposition interpretiert wurde (EgmondFröhlich et al., 2007), ist vor allem im Hinblick auf die altersdifferenzierte Ausweisung mittlerer Effektstärken interessant. Hier zeigen Marshall et al., dass Medieneffekte auf Körperfettgehalt am höchsten im Vor- und Grundschulalter sind $\left(r_{\mathrm{c}}=0.19 \mathrm{im}\right.$ Alter von $0-6$ Jahren beziehungsweise $\mathrm{r}_{\mathrm{c}}=0.13 \mathrm{im}$ Alter von 7 - 12 Jahren), während sie bei Jugendlichen nicht oder kaum messbar sind. Ein umgekehrter Zusammenhang zeigt sich zwischen Mediennutzung, Alter und physischer Aktivität. Während bei jugendlichen Stichproben ein solcher $\mathrm{Zu}$ sammenhang durch die Meta-Analyse bestätigt wird $\left(r_{\mathrm{c}}=-0.15\right.$ im Alter von $13-$ 18 Jahren), sind die Zusammenhänge bei jüngeren Stichproben deutlich schwächer $\left(r_{\mathrm{c}}=-0.12 \mathrm{im}\right.$ Alter von $7-12$ Jahren; $r_{\mathrm{c}}=-0.06$ im Alter von 0 - 6 Jahren). Insgesamt, so folgern Marshall et al., reiche allein der Konsum elektronischer Medien kaum aus, um Übergewichtigkeit in einem klinisch relevanten $\mathrm{Maß}$ zu steigern (S. 1241). Erst in Verbindung mit anderen Faktoren und unter Berücksichtigung der Tatsache, dass allein die Schule Kinder und Jugendliche täglich mehrere Stunden lang zum Stillsitzen veranlasse, komme Medienkonsum eine Bedeutung für Übergewichtigkeit zu.

Trotz der kleinen Effekte und Unsicherheiten bei der Beurteilung der genauen Wirkmechanismen verweisen die Autoren aber darauf, dass es deutliche An-

Zusammenhangsstudien zumeist durch Messung der Hautfaltendicke erhoben. Aufgrund der hohen Korrelation zwischen Hautfaltendicke und Body-Mass-Index (BMI) (vgl. Robinson, 1999, S. 1562), kann auch der BMI selbst als recht guter indirekter Indikator für den Körperfettgehalt gelten. $60 \%$ der von Marshall et al. analysierten Studien zum Körperfettgehalt verwendeten die Hautfaltendicke als primären Indikator, $37 \%$ die Messung des BMI durch Gewichts- und Größenmessung, $3 \%$ verwendeten entweder selbst berichtete Größen- und Gewichtsangaben zur BMI-Berechung oder direkte klinische Strahlungsmessverfahren (1 Studie).

75 Als Maß für die mittlere Effektstärke verwendeten die Autoren eine korrigierte Form von Pearsons $r$ : Die vollständig korrigierte am Untersuchungssample korrigierte mittlere Effektstärke rc (Marshall et al., 2004, S. 1239). 
zeichen für eine kausale Korrelation zwischen Mediennutzung, Übergewicht und Bewegungsarmut gebe (Marshall et al., 2004, S. 1242). Dabei verweisen sie auf die signifikante übergewichtsreduzierende und bewegungsfördernde Wirkung von Interventionsprogrammen (Epstein, Leonard H., Paluch, Gordy \& Dorn, 2000; Gortmaker, Steven L. et al., 1999; Robinson, 1999), in denen die Menge der Fernseh- und Computerspielnutzung reduziert wurde.

Bei der Frage nach genauen Wirkmechanismen von Mediennutzung auf Übergewichtigkeit wird einerseits der mit ausdauerndem Medienkonsum assoziierte Bewegungsmangel ins Feld geführt, andererseits wird auf die ungünstige Wirkung medienvermittelter Werbung für kalorienreiche, ansonsten aber nährwertarme Snacks und Nahrungsmittel verwiesen. Insofern gilt es zwischen möglichen Effekten der Fernsehnutzung und der Nutzung interaktiver Computerspiele deutlich zu differenzieren, da Bewegungsmangel eine Folge beider Tätigkeiten sein kann, während ernährungsbezogene Werbebotschaften im Rahmen von Computerspielen derzeit (noch) keine Rolle spielen. Da viele diesbezügliche Studien häufig lediglich die mit Fernsehen oder elektronischen Medien insgesamt verbrachte Zeit messen, können solche Studien zunächst wenig über die genaue Art des Zusammenhangs zwischen Mediennutzung und Übergewichtigkeit aussagen.

\subsubsection{Wirkmechanismen: Werbung}

Dass Werbung tatsächlich einen Einfluss auf die Produktwahrnehmung und das Konsumverhalten von Kindern hat, zeigten Desmond und Carveth (2007) in einer Meta-Analyse zur Wirkung von Werbung auf Kinder und Jugendliche. Die berichteten Effekte wurden von den Autoren allerdings als schwach eingestuft (S. 176). So fanden sie im Rahmen ihrer Analyse von 14 Studien mit voneinander unabhängigen Stichproben mit $\mathrm{N}=4.253$ Probanden einen durchschnittlichen Effekt von $r=0.14$ der Werbenutzung auf (positivere) Einstellungen zu den beworbenen Marken (S. 175). Darüber hinaus identifizierten Desmond und Carveth sechs Studien mit voneinander unabhängigen Stichproben mit insgesamt $\mathrm{N}=1.297$ Probanden zur Frage, inwieweit Werbung konkrete Verhaltenseffekte auf Kinder und Jugendliche hat. Hier fanden sie einen durchschnittlichen Effekt von $r=0.155$. Das Resümee der Autoren, die signifikante, aber schwache Korrelation zwischen Werberezeption, Einstellungen und Verhalten lasse auf keine praktischen Auswirkungen der Werbung schließen (S. 176), erscheint allerdings, zumindest im Hinblick auf das Ernährungsverhalten von Kindern, fragwürdig, befand sich im Analyse-Sample der Studie nur 
ein Experiment (Dawson, Jeffrey \& Walsh, 1988) zu Werbeeffekten auf das Ernährungsverhalten von Kindern. Umfangreiche systematische Literaturreviews von Hastings et al. (2003), Hastings, Gerard, McDermott, Angus, Stead und Thomson (2007) sowie Livingstone (2005) zur allgemeinen Werbewirkung auf die Ernährung von Kindern und Reviews zur speziellen Wirkung von TVWerbung auf kindliches Ernährungsverhalten (Kunkel et al., 2004; McGinnis, Gootman \& Kraak, 2005) belegen dagegen eindeutige und relevante Effekte der Werbung insbesondere für kalorienreiche Produkte auf das Ernährungsverhalten von Kindern und Jugendlichen. Hastings et al. fassen die Studienlage zu Werbeeffekten folgendermaßen zusammen:

„Evidence from more complex studies (capable of establishing causality) shows that this promotional activity is having an effect on children. [...] food promotion influences children's food preferences, and encourages them to ask their parents to purchase foods they have seen advertised. Food promotion has also been shown to influence children's consumption and other diet-related behaviours and outcomes. These effects are significant, independent of other influences and operate at both brand and category level." (Hastings, Gerard et al., 2007, S. 2).

Livingstone und Helsper warnen in diesem Kontext vor der Annahme, die stärksten Werbeffekte seien bei den jüngsten Kindern zu vermuten. Vielmehr habe sich die Werbung den je nach Alter der Kinder unterschiedlichen Kompetenzen im Umgang mit persuasiver Kommunikation angepasst und sei in allen Altersstufen ungefähr gleich wirksam (2006, S. 576). Würden jüngere Kinder mit geringerer Werbekompetenz unter Zuhilfenahme attraktiver oder prominenter Akteure, Jingles und farbenfroher Bildsprache angesprochen, sei Werbung für ältere Kinder und Teenager eher argumentativ gestaltet und enthalte bereits Antworten auf bekannte Gegenargumente (ebenda).

\subsubsection{Wirkmechanismen: Bewegungsmangel}

Dass neben der in den Medien rezipierten Werbung auch Bewegungsmangel während der Mediennutzung eine Ursache für Übergewichtigkeit sein kann, zeigen Studien, in denen Mediennutzungszeiten und genutzte Medieninhalte nicht (zum Beispiel durch alleinige Abfrage der durchschnittlichen Fernsehnutzungszeiten) untrennbar konfundiert sind. Myrtek und Scharff (2000) belegten mithilfe einer physiologischen Monitoring-Studie ${ }^{76}$ an $\mathrm{N}=100$ elf- und

76 Dabei wurden sämtliche Untersuchungsteilnehmer für 23 Stunden mit einem Minicomputer ausgestattet, der mithilfe entsprechender Sensoren kontinuierlich Herzfrequenz 
N=100 15-jährigen Schülerinnen und Schülern in Süddeutschland, dass Vielseher deutlich geringe körperliche Aktivitäten im Laufe eines Tages aufweisen und insbesondere stärkere Belastungen (Treppen steigen, Fahrrad fahren) meiden. Die Schweizer Querschnittsstudie von Stettler, Signer und Suter (2004) an $\mathrm{N}=872$ Kindern erster bis dritter Klassen belegte darüber hinaus einen $\mathrm{Zu}$ sammenhang zwischen Computerspielnutzung und Übergewichtigkeit unter statistischer Kontrolle der Fernsehnutzung und weiterer wichtiger soziodemografischer Merkmale der Schülerinnen und Schüler, was letztlich für einen eigenständigen Effekt der medieninduzierten Bewegungsarmut auf Übergewichtigkeit spricht.

\subsubsection{Essstörungen}

Der - wenn auch geringe - Zusammenhang zwischen Mediennutzung und Übergewichtigkeit erscheint manchem insofern paradox, als das in den Medien präsentierte Schönheitsideal ein vollkommen anderes ist. Das insbesondere durch Fernsehen und Zeitschriften verstärkte Schönheitsideal ist das einer - aus medizinischer Sicht - untergewichtigen Frau beziehungsweise eines schlanken, unrealistisch muskelstrotzenden Mannes (eine Zusammenfassung einiger Befunde bei Groesz, Levine \& Murnen, 2002, S. 2). Und so finden sich neben den oben beschriebenen Medieneffekten auf die Entwicklung einer Übergewichtigkeit in der Literatur ebenso Hinweise, dass auch Essstörungen wie Magersucht oder Bulimie einen Zusammenhang mit bestimmten Mediennutzungsmustern aufweisen. Stice, Schupak-Neuberg, Shaw \& Stein (1994) fanden im Rahmen einer Befragungsstudie an $\mathrm{N}=238$ Psychologiestudentinnen erstmals einen direkten schwachen bis mittleren Zusammenhang zwischen der Intensität der Zeitschriften- und TV-Nutzung ${ }^{77}$ und der Häufung anorexie- und bulimieassoziierter Symptome. Über die kausale Richtung dieses Zusammenhangs sagt die gefundene Korrelation freilich nichts aus, zumal der Auflösungsgrad der Mediennutzung äußerst beschränkt war.

und Bewegungsaktivität aufzeichnete und auswertete (Myrtek, Foerster \& Brügner, 2001).

77 Im verwendeten Intensitätsindex der Mediennutzung wurde die Menge der im letzten Monat genutzten Zeitschriften der Genres Gesundheit und Fitness, Beauty und Mode, Unterhaltung, Kunst und Klatsch mit der Anzahl der Stunden verrechnet, die im letzten Monat für die Rezeption für TV-Comedy, TV-Drama und TV-Gameshows aufgewendet wurde (Stice et al., 1994, S. 837). 
Einen weiteren Hinweis auf einen Zusammenhang zwischen Mediennutzung und gestörtem Essverhalten brachte eine Studie in Nadroga (Fiji), wo erst im Jahr 1995 das Fernsehen eingeführt wurde (Becker, Burwell \& Gilman, 2002). Mithilfe von zwei Querschnittsmessungen (möglicherweise gestörten) Essverhaltens aller Schülerinnen fünfter bis siebter Klassen in Nadroga vor $(\mathrm{N}=63)$ und drei Jahre nach Einführung des Fernsehens $(\mathrm{N}=65)$ konnten Aussagen darüber gemacht werden, inwieweit sich das traditionell sehr wenig problematische Essverhalten der jugendlichen Inselbewohnerinnen nach Einführung des Fernsehens änderte. Tatsächlich wiesen die Autoren der Studie einen signifikanten Anstieg gestörten, insbesondere bulimischen Verhaltens junger Mädchen nach Einführung des Fernsehens nach. Insgesamt betrachtet sind die beiden dargestellten Studien jedoch die einzigen Studien, die einen direkten $\mathrm{Zu}$ sammenhang zwischen bulimisch-anorektischen Essstörungen herstellen, insgesamt gilt die diesbezügliche Studienlage als äußerst lückenhaft (Harrison \& Cantor, 1997).

Als wenig strittig gilt indessen der Befund, dass Fernsehnutzung und insbesondere Zeitschriftennutzung im Zusammenhang mit der Internalisierung medienvermittelter geschlechtstypischer Schönheitsideale steht und daraus folgend $\mathrm{zu}$ einer größeren Unzufriedenheit mit dem eigenen Körper führt. Insbesondere Mädchen erscheinen anfällig für die Übernahme unrealistischer (dünner) Schönheitsideale, da Mädchen bereits frühzeitig vermittelt wird, ihren Körper zu gebrauchen, um anderen zu gefallen (Stephens, Hill \& Hanson, 1994). In einer Meta-Analyse von 25 experimentellen Studien mit 43 unabhängigen Stichproben mit an $\mathrm{N}=2.292$ Probandinnen untersuchten Groesz, Levine und Murnen (2002) die Effekte der medialen Präsentation dünner Schönheitsideale auf die Zufriedenheit von Mädchen und jungen Frauen mit ihrem eigenen Körper. Dabei fanden die Autoren einen mittleren Effekt von Cohen's $d=-0.31$ der medialen Präsentation auf die Körperzufriedenheit der Frauen (S. 11). Bemerkenswert erscheint in diesem Zusammenhang, dass die Analyse von Groesz, Levine und Murnen diese Effekte bereits bei Mädchen im vorpubertären Alter fand. Neuere Befragungsstudien zum Zusammenhang zwischen alltäglicher Mediennutzung und der Wahrnehmung des eigenen Körpers konnten dies für acht- bis elfjährige Mädchen (Jung \& Peterson, 2007) beziehungsweise neun- bis zwölfjährige Mädchen (Clark \& Tiggemann, 2006) erneut bestätigen, wobei Clark und Tiggemann zeigten, dass der Mediennutzung kein direkter Einfluss auf Selbstwahrnehmung und Körperzufriedenheit zukommt, sondern nur indirekte Medieneffekte vermittels der Kommunikation mit Peers zu beobachten sind (S. 638 - 639). Baumann (2009) bemerkt völlig zu Recht, dass die Entwicklung einer Essstörung Resultat eines komplexen Gefüges individueller Faktoren in 
Interaktion mit komplexen Umweltbedingungen ist. White (1992) zeigt, dass biologisch-genetische, psychologische, familiäre und soziokulturelle Faktoren die Entstehung einer Essstörung bedingen (wobei die Medien nur ein Faktor unter vielen weiteren soziokulturellen Faktoren sind). Und doch zeigt sich insbesondere bei weiblichen Jugendlichen und auch bereits bei Kindern, dass Medien ein Verstärkungsfaktor solcher Störungen sein können.

\subsubsection{Fazit zu Medienwirkungen auf physische Parameter}

Es konnte gezeigt werden, dass die Nutzung elektronischer Medien bei Kindern und Jugendlichen - insbesondere in der pädiatrischen Literatur - mit einer Reihe gesundheitlicher Probleme assoziiert wird. Neben aus der Arbeitswelt bekannten Phänomenen wie muskuloskeletalen Beschwerden, Augenbeschwerden und Kopfschmerzen belegen eine Reihe von Studien einen Zusammenhang von elektronischer Mediennutzung mit Übergewichtigkeit, Bewegungsarmut, gestörtem Essverhalten und Schlafstörungen. Das Gros der Studien bleibt aufgrund ihrer Querschnittsanlage allerdings einen eindeutigen Kausalitätsnachweis schuldig. Wo elaborierte Studien zur Kausalität dieser Zusammenhänge vorliegen, zeigt sich in der Regel ein sehr schwacher direkter Effekt der elektronischen Mediennutzung (zum Beispiel bei der Forschung zu Übergewichtigkeit und Essstörungen), so dass davon auszugehen ist, dass die genannten medienassoziierten Probleme das Resultat eines komplexen Gefüges individueller Faktoren in Interaktion mit komplexen Umweltbedingungen sind. Elektronische Medien dürften hier in der Regel eher als Problemverstärker denn als Hauptursache wirken.

\subsection{Computerspielabhängigkeit}

Ein Forschungsgegenstand, der mit der zunehmenden Verbreitung interaktiver, multimedialer Unterhaltungsmedien immer stärker in den Vordergrund getreten ist, ist der Aspekt suchtartiger Mediennutzung. Während Young, Pistner, O'Mara und Buchanan (1999) auf der Grundlage einer onlinegestützten Befragung von amerikanischen Psychotherapeuten fünf verschiedene Erscheinungsformen suchtartiger Mediennutzung ${ }^{78}$, so genannte Cyber-Disorders, identifiziert haben,

78 Young et al. unterscheiden zwischen Cybersexual Addiction (Abhängigkeit von pornografischen Angeboten im Internet), Cyber-relational Addiction (übermäßige Pflege von 
erscheint im Hinblick auf die Mediennutzung von Kindern und Jugendlichen die Computerspielabhängigkeit von besonderer Relevanz und wurde in den letzten Jahren bei weitem am intensivsten untersucht (ein Überblick zum nationalen und internationalen Forschungsstand bei Rehbein et al., 2009a). Ist zeitlich exzessives Computerspielen von Kindern und Jugendlichen auch ein bereits länger diskutiertes Phänomen ${ }^{79}$, standen bisher eher die Folgen eines solchen Spielverhaltens im Fokus. Der Frage, ob zeitlich exzessives Computerspielen - zunächst unabhängig von vermuteten Wirkungen - ein auch von den Betroffenen wahrgenommenes, eigenständiges Problem darstellen kann, wurde erst in Studien der letzten zehn Jahren nachgegangen.

\subsubsection{Theoretischer Hintergrund}

Studien von Yang (2001), Hauge und Gentile (2003), Grüsser, Thalemann, Albrecht und Thalemann (2005), Wölfling, Thalemann und Grüsser (2007), sowie Studien des Kriminologischen Forschungsinstituts Niedersachsen (Baier \& Rehbein, 2009; Rehbein et al., 2009a) kommen zu dem Schluss, dass bei einem Teil jugendlicher Exzessivspieler/-innen Verhaltensmuster vorliegen, die mit einer stoffgebundenen Abhängigkeit nach Maßgabe internationaler Diagnosekriterien des ICD-10 vergleichbar sind ${ }^{80}$. Betroffene Jugendliche berichten von

Onlinebeziehungen), Net Compulsions (abhängiges Glückspiel, Kaufen und Handeln im Internet), Information Overload (abhängiges Surfen oder Absuchen von Datenbanken) sowie Computer Addiction (inkl. abhängiges Computerspielen).

79 Zur Problematik schlechterer Schulleistungen infolge von Time-Displacement-Effekten durch zeitlich exzessives Computerspielen vgl. Mößle et al. (2007) sowie S. 62 bis 81 in dieser Arbeit. Zur möglichen Problematik mangelnder sozialer Kompetenzen als Folge zeitlich exzessiven Computerspielens vgl. Mößle und Kleimann (2009), Kleimann und Mößle (2008) sowie Fußnote 69 in dieser Arbeit.

80 In der zehnten Revision der internationalen statistischen Klassifikation der Krankheiten und verwandter Gesundheitsprobleme (ICD-10, German Modification, Version 2010, Kapitel V, Psychische und Verhaltensstörungen, F12) wird das Abhängigkeitssyndrom beschrieben als eine „Gruppe von Verhaltens-, kognitiven und körperlichen Phänomenen, die sich nach wiederholtem Substanzgebrauch entwickeln. Typischerweise besteht ein starker Wunsch, die Substanz einzunehmen, Schwierigkeiten, den Konsum zu kontrollieren, und anhaltender Substanzgebrauch trotz schädlicher Folgen. Dem Substanzgebrauch wird Vorrang vor anderen Aktivitäten und Verpflichtungen gegeben. Es entwickelt sich eine Toleranzerhöhung und manchmal ein körperliches Entzugssyndrom.“ (Deutsches Institut für Medizinische Dokumentation und Information, 2009; eine ausführliche Erläuterung bei Rehbein \& Borchers, 2009) 
einem starken Verlangen ${ }^{81}$ danach, Computerspiele $\mathrm{zu}$ nutzen, von $\mathrm{zu}-$ nehmendem Kontrollverlust über das eigene Spielverhalten, zunehmender Fokussierung des eigenen Denkens und Handelns auf Herbeiführung und Aufrechterhaltung der Spielsituation, von der Notwendigkeit immer längerer Spielzeiten zur Herbeiführung einer Befriedigung des Spielverlangens, einer Fortsetzung des Computerspielens trotz bereits erfolgter negativer sozialer oder beruflich-schulischer Konsequenzen sowie regelrechten Entzugserscheinungen bei längerer Spielabstinenz.

Das Konzept nicht-stoffgebundener Verhaltenssüchte wurde bereits seit Ende des 19. Jahrhunderts diskutiert (Grüsser \& Thalemann, 2006), fand jedoch erst im Form des Pathologischen Glückspielens Eingang in die Klassifikationen psychischer Störungen (ein Überblick zur Erforschung und Diagnostik von Verhaltenssüchten bei Grüsser, Poppelreuter, Heinz, Albrecht \& Saß, 2007; sowie bei Wölfling, Müller \& Beutel, 2009). Da Pathologisches Glücksspiel als Impulskontrollstörung klassifiziert wird, ist das allgemeine Konzept der Verhaltenssüchte ebenso wie das spezifische Konzept der Computerspielabhängigkeit derzeit nicht offiziell anerkannt, obwohl eine angemessene Diagnostik und Behandlung suchtartiger Verhaltensweisen dadurch deutlich erschwert wird (Grüsser et al., 2007; Rehbein et al., 2009a; Wölfling et al., 2009) und die theoretische Ausarbeitung des Verhaltenssuchtkonzept inzwischen äußerst elaboriert ist. So zeigten Grüsser et al. (2007) unter Berufung auf den derzeitigen internationalen Forschungsstand, dass bei der Entstehung und Aufrechterhaltung einer Verhaltenssucht psychologischen ebenso wie neurobiologischen Faktoren eine große Bedeutung zukommt und postuliert einen integrativen Ansatz aus lerntheoretischen und neurobiologischen Befunden zur Erklärung der Entstehung von Verhaltenssüchten wie der Computerspielabhängigkeit (S. 1001). Demnach sei die Erinnerung an die positive Suchtmittelwirkung der zentrale Motivator für das süchtige Verhalten. Die Ausübung von süchtigem Verhalten sei auch bei substanzungebundener Verhaltenssucht erlernt, wodurch Lernprozessen, insbesondere der klassischen und operanten Konditionierung eine entscheidende Rolle sowohl bei der Entstehung wie Aufrechterhaltung einer Sucht zukomme (ebenda). Zuvor neutrale Reize (zum Beispiel externale Stimuli wie der Anblick eines Spielautomaten oder internale Reize wie bestimmte Gefühlszustände oder Stresssituationen) werden demnach mit dem Suchtverhalten oder der Sucht-

81 Starkes Verlangen (Craving) ist allerdings im Hinblick auf die Diagnostik von Computerspielabhängigkeit als problematisch kritisiert worden und wird daher häufig nicht als zentrales Kennzeichen einer Computerspielabhängigkeit gesehen (Rehbein \& Borchers, 2009; Rehbein et al., 2009a). 
mittelwirkung assoziiert und lösen so als erlernte (konditionierte) Reize einen motivationalen Zustand (Suchtmittelverlangen) und eine erlernte (konditionierte) Reaktion (Suchtmittelgebrauch) aus (ebenda). Die Erinnerung an den ,erfolgreichen" Suchtmittelgebrauch trage dann nach den Prinzipien der operanten Konditionierung zur weiteren Verstärkung und Wiederholung des Suchtverhaltens bei (ebenda).

Nach Grüsser et al. wird bei der Verhaltenssucht ähnlich wie bei der stoffgebundenen Abhängigkeit dem verhaltensverstärkenden Belohnungssystem eine zentrale Rolle zugeschrieben. Innerhalb dieses Belohnungssystems finde sich ein hoch komplexes Zusammenspiel bestimmter Neurotransmitter wie Dopamin, Serotonin sowie verhaltensmodulierender Neuropeptide wie auch lernrelevanter wichtiger Botenstoffe aus dem glutamatergen System, die in den letzten Jahren immer besser als physiologische Korrelate der lerntheoretischen Suchtentstehungsmodelle identifiziert werden konnten (ebenda). Griffiths (2008) sowie Rehbein et al. (2009a) argumentieren nun, dass sich in bestimmten Computerspielen, insbesondere Online-Rollenspielen, spielstrukturelle Merkmale finden, die mithilfe operanter Konditionierungsprozesse und insbesondere intermittierender Verstärkungsprozesse (einer besonderen Spielart der operanten Konditionierung, innerhalb derer bestimmtes Verhalten nur unregelmäßig verstärkt wird) Spielmotivation weiter steigern und suchtartiges Spielverhalten zusätzlich intensiveren. Diese Verstärkungen erfolgen in Computerspielen mithilfe ausgefeilter Belohnungssysteme. Die Kombination verschiedener belohnender Elemente (etwa durch Vergabe von Punkten, Ranglistenplatzierungen, frei gespielter Ausrüstungsgegenstände, neuer Fähigkeiten oder Gestaltungsmöglichkeiten der eigenen Spielfigur) und die Variation der Belohnungsfrequenz erhöht den Spielanreiz und die Spielgratifikation. Zudem erfolgt in Multi-PlayerSpielen eine zusätzliche Belohnungsverstärkung durch Prestige- und Einflussgewinn in der Spiel-Community, womit dem Computerspielen vielfach auch eine identitätsstiftende Bedeutung für den Spieler zukommt (ebenda). Es erscheint daher nur folgerichtig, dass insbesondere unter Spielern von Massive Multiplayer Online Roleplay Games (MMORPGs), also Rollenspielen, die mithilfe von per Internet vernetzten Computern von vielen hundert oder tausend Spielern gleichzeitig gespielt werden, aufgrund der Kombination aller oben genannten Spielmerkmale eine signifikante Häufung suchtartigen Computerspielverhaltens zu finden ist.

Rehbein, Kleimann und Mößle zeigten, dass unter Spielern des weltweit erfolgreichsten MMORPGs World of Warcraft der Anteil von als computerspielabhängig oder abhängigkeitsgefährdet eingestuften männlichen Jugendlichen mit 20,1 Prozent fast dreimal so hoch lag, wie unter dem Durchschnitt männlicher 
jugendlicher Computerspieler (Anteil abhängiger oder abhängigkeitsgefährdeter Spieler hier: 7,7\%) (Rehbein et al., 2009a, S. 145). Selbst unter statistischer Kontrolle weiterer eine Abhängigkeit begünstigender Faktoren (vgl. Rehbein et al., 2009b, S. 29) zeigte sich ein stabiler signifikanter Effekt der Nutzung von MMORPGs auf die Entstehung einer Computerspielabhängigkeit.

\subsubsection{Prävalenzzahlen}

\subsubsection{Computerspielabhängigkeit bei Jugendlichen und jungen Erwachsenen}

Derzeit sind nur wenige verlässliche Studien zur Prävalenz zur Computerspielabhängigkeit verfügbar, wobei sich fast alle publizierten Studien auf Computerspielerinnen und -spieler im Jugendalter beziehen. Die Spannbreite bisher veröffentlichter Prävalenzzahlen zur Computerspielabhängigkeit unter Jugendlichen reicht dabei von 5 bis 15 Prozent (Rehbein \& Borchers, 2009, S. 43 - 44). Einerseits hängt dies mit der Uneinheitlichkeit diagnostischer Standards zusammen, andererseits arbeiten die meisten bisher veröffentlichten Studien mit anfallenden Stichproben.

Die „Pathologisierung“ des Computerspielens durch teilweise zweistellige Abhängigkeitsprävalenzen ist verschiedentlich kritisiert worden (Griffiths, Mark D., 2009; Rehbein \& Borchers, 2009; Rehbein et al., 2009a). So wird in einigen Studien beispielsweise die reine mit Computerspielen verbrachte Zeit ab einer bestimmen Menge als ein Abhängigkeitskriterium definiert, was eine Abgrenzung zwischen leidenschaftlichem, aber ansonsten zunächst unproblematischem Spielverhalten und suchtartigem Verhalten deutlich erschwert. Anderseits wurden teilweise Abhängigkeitskriterien des amerikanischen Diagnosemanuals DSM-IV ${ }^{82}$ weitgehend unverändert auf die Computerspiel-

82 Im DSM-IV-Manual (Diagnostic and Statistical Manual of Mental Disorders DSM-IVText Revision) der American Psychiatric Association werden die Kriterien einer stoffgebundenen Abhängigkeit folgendermaßen definiert:

A maladaptive pattern of substance use, leading to clinically significant impairment or distress, as manifested by three (or more) of the following, occurring at any time in the same 12-month period:

(1) tolerance, as defined by either of the following: (a) a need for markedly increased amounts of the substance to achieve Intoxication or desired effect (b) markedly diminished effect with continued use of the same amount of the substance

(2) Withdrawal, as manifested by either of the following: (a) the characteristic withdrawal syndrome for the substance (refer to Criteria A and B of the criteria sets for 
nutzung übertragen, ohne sie dem Forschungsgegenstand angemessen zu gewichten. Diesen Überlegungen wird inzwischen in neueren Erhebungsinstrumenten Rechnung getragen (Meerkerk, Van Den Eijnden, Vermulst \& Garretsen, 2009; Rehbein et al., 2009a). Landesweit repräsentative Daten stehen bisher nur für 15-jährige Jugendliche in Deutschland (Rehbein et al., 2009a) sowie für acht- bis achtzehnjährige US-amerikanische Kinder beziehungsweise Jugendliche (Gentile, 2009) zur Verfügung.

Mithilfe einer schriftlichen Befragung von $\mathrm{N}=15.168$ Schülerinnen und Schüler neunter Klassen (Durchschnittsalter: 15,3 Jahre) ermittelten Rehbein, Kleimann und Mößle deutschlandweit repräsentative Prävalenzdaten für diese Altersgruppe von 1,7 Prozent computerspielabhängigen Jugendlichen. Weitere 2,8 Prozent wurden als computerspielabhängigkeitsgefährdet eingestuft. Basierend auf der eigens entwickelten Computerspielabhängigkeitsskala KFNCSAS-II, die sich eng an die Suchtklassifikation des ICD-10 anlehnt, wurden dabei insbesondere männliche Jugendliche als anfällig für eine Computerspielabhängigkeit identifiziert. So wurden 3 Prozent Jungen, aber nur 0,3 Prozent der Mädchen als computerspielabhängig klassifiziert. Weitere 4,7 Prozent der Jungen und nur 0,5 Prozent der Mädchen wurden als gefährdet eingestuft (Rehbein et al., 2009a, S. 143 - 144).

Withdrawal from the specific substances) (b) the same (or a closely related) substance is taken to relieve or avoid withdrawal symptoms

(3) the substance is often taken in larger amounts or over a longer period than was intended

(4) there is a persistent desire or unsuccessful efforts to cut down or control substance use

(5) a great deal of time is spent in activities necessary to obtain the substance (e.g., visiting multiple doctors or driving long distances), use the substance (e.g., chainsmoking), or recover from its effects

(6) important social, occupational, or recreational activities are given up or reduced because of substance use

(7) the substance use is continued despite knowledge of having a persistent or recurrent physical or psychological problem that is likely to have been caused or exacerbated by the substance (e.g., current cocaine use despite recognition of cocaine-induced depression, or continued drinking despite recognition that an ulcer was made worse by alcohol consumption)

(American Psychiatric Association, 2001, Kursivsetzung durch den Autor) 
Gentile (2009) legte eine landesweit nach eigenen Angaben repräsentative ${ }^{83}$ Online-Befragung von $\mathrm{N}=1.178$ US-amerikanischen Minderjährigen im Alter von 8 bis 18 Jahren vor, mit deren Hilfe er eine Computerspielabhängigkeitsprävalenz ${ }^{84}$ unter Computerspielerinnen und Computerspielern von 8,5 Prozent schätzt. Dieser Wert ist im Vergleich zu den Daten von Rehbein et al. (2009a) deutlich höher, kommt letztere Studie selbst unter computerspielenden Jungen (die auch in der Studie von Gentile deutlich höhere Belastungswerte aufweisen) nur auf 7,7 Prozent abhängige und gefährdete Computerspieler. Diese hohe Prävalenz ist jedoch unter mehreren Gesichtspunkten anzuzweifeln ${ }^{85}$ und bedarf einer dringenden Revision.

Obwohl Prävalenzwerte einzelner Studien mit einiger Sicherheit als zu hoch erscheinen, um ein ernsthaft pathologisches Geschehen zu beschreiben, und obwohl kaum repräsentative landesweite Prävalenzdaten vorliegen, lässt sich dennoch bereits feststellen, dass Computerspielabhängigkeit ein ernstzunehmendes Problem mit ernsthaften Folgen darstellt. So zeigten Rehbein et al. (2009a), dass männliche computerspielabhängige Jugendliche Leistungseinbrüche in der Schule aufweisen, dass sie aufgrund ihrer Abhängigkeit deutlich häufiger der Schule fernbleiben und dass sie neben dem Computerspielen kaum einer regelmäßigen realweltlichen Freizeitbeschäftigung nachgehen (S. 144).

$83 \mathrm{Zu}$ diesem Zweck wurde im Online-Panel des Markt- und Meinungsforschungsinstitutes Harris-Interactive eine altersgeschichtete Stichprobe gezogen. Ob es sich beim OnlinePanel selbst um zufällig ausgewählte Personen oder um eine Quota-Stichprobe handelt, ist der Publikation nicht zu entnehmen. Auch die Tatsache, dass der Besitz eines Internetanschlusses Grundvoraussetzung für eine Befragungsteilnahme ist, wird nicht weiter diskutiert, obwohl diese Tatsache beim konkreten Forschungsgegenstand „Computerspielabhängigkeit" von nicht unerheblicher Bedeutung ist.

84 Gentile selbst verwendet zumeist die Begrifflichkeit pathological video game use, spricht aber immer wieder auch von addiction und verwendet bei Konstruktion seiner Skala für pathological video game use die DSM-IV-Kriterien für stoffgebundene Süchte.

85 Obwohl kulturspezifische Unterschiede in der Computerspielnutzung zwischen den USA und Deutschland hier natürlich auch eine Rolle spielen können, lässt sich der Unterschied wahrscheinlich vor allem mit einer deutlich konservativeren (und exakteren) Diagnostik in der deutschen Studie erklären, in der beispielsweise das Craving nicht als typisches Merkmal einer Computerspielabhängigkeit operationalisiert wird, in der aufgrund ihrer Konstruktion bestimmte Kernkriterien (wie beispielsweise das Auftreten negativer Konsequenzen) zwingend erfüllt sein müssen und die eine differenzierte Abstufung des Auftretens von Abhängigkeitskriterien ermöglicht. Noch ein weiteres Ergebnis von Gentile spricht gegen die Tatsache, dass hier tatsächlich Computerspielabhängigkeit gemessen wurde: Obwohl aufgrund theoretischer Überlegungen als auch aufgrund empirischer Befunde (vgl. Mößle \& Rehbein, 2008; Rehbein et al., 2009b) davon ausgegangen werden muss, dass die Prävalenz von Computerspielabhängigkeit zumindest heutzutage in der Adoleszenz die höchste Auftretenswahrscheinlichkeit hat, berichtet Gentile, es gäbe keinen Zusammenhang zwischen Computerspielabhängigkeit und Alter. 
Zudem weisen diese Jugendlichen geringere Schlafzeiten und eine erhöhte psychische Belastung auf (ebenda). Mößle und Rehbein (2008) wiesen darüber hinaus an einem Sample junger Erwachsener Berufsschüler und Gymnasiasten einen hohen Leidensdruck von computerspielabhängigen Personen hinsichtlich gesundheits- und leistungsbezogener sowie sozialer Folgen des abhängigen Computerspielens nach. Zugleich fanden sich weder in dieser Studie noch in der deutschlandrepräsentativen Schülerstudie Belege dafür, dass Computerspielabhängigkeit lediglich das Symptom einer anderen zugrundeliegenden psychischen Erkrankung darstellen könnte (Rehbein et al., 2009b, S. 42). Einer Depressions-, oder Angsterkrankung kam unter Berücksichtigung subklinischer Merkmale ebenso keine Relevanz für die Vorhersage von Computerspielabhängigkeit im Jugend- und Erwachsenenalter zu, wie auch andere Suchterkrankungen keine besonders auffälligen Komorbiditäten zu Computerspielabhängigkeit aufwiesen (Mößle \& Rehbein, 2008).

\subsubsection{Computerspielabhängigkeit im Kindesalter}

Im Vergleich zu Untersuchungen über Computerspielabhängigkeit unter Jugendlichen und jungen Erwachsenen ist die Studienlage zu Computerspielabhängigkeit im Kindesalter noch einmal deutlich defizitärer. Zwar untersuchte Gentile (2009) im Rahmen seiner Erhebung pathologisches Computerspielverhalten von 8 - 18-jährigen Kindern und Jugendlichen in den USA, weist in seiner Darstellung der Ergebnisse jedoch keine altersdifferenzierten Prävalenzen pathologischer Computerspielnutzung aus (vgl. Fußnote 85).

Im deutschsprachigen Raum liegen derzeit zwei Studien an Berliner Grundschülerinnen und Grundschülern vor. Einerseits untersuchte die Forschungsgruppe um Grüsser und Thalemann eine anfallende Stichprobe von $\mathrm{N}=323$ Kindern der sechsten Grundschulklasse ${ }^{86}$ (Durchschnittsalter: 11,8 Jahre) im Hinblick auf ihr Computerspielverhalten (Grüsser et al., 2005; Thalemann, Albrecht, Thalemann \& Grüsser, 2004). Anderseits publizierten Rehbein, Kleimann und Mößle (2009b, S. 30 - 40) Ergebnisse ihrer Untersuchung der Computerspielnutzung von 1.156 für Berlin repräsentativ ausgewählten Grundschülern der fünften Grundschulklassen (Durchschnittsalter: 11,4 Jahre).

86 Im Gegensatz zu den meisten anderen deutschen Bundesländern reicht in Berlin der Grundschulbesuch von der ersten bis zur sechsten Klasse. Eine Ausnahme bildet der Besuch eines Gymnasiums mit altsprachlichem Bildungsgang, der bereits nach Abschluss der vierten Klasse beginnt. 
Grüsser et al. (2005) berichten von 9,3 Prozent der Schülerinnen und Schüler, die ein exzessives Computerspielverhalten aufweisen. Dabei seien Jungen mit 13,1 Prozent exzessiver Computerspieler deutlich häufiger betroffen als Mädchen (4,7\%). Obwohl innerhalb des Klassifikationsinstruments zur exzessiven Computerspielnutzung unter anderem Abhängigkeitsmerkmale nach ICD-10-Standard abgefragt wurden, weisen die Autoren darauf hin, ,dass für das Kindesalter [...] Formulierungen wie ,nichtstoffgebundene Abhängigkeit“ jedoch nur mit Vorsicht verwendet werden [sollten] (S. 193) und wählen insofern durchgängig den Terminus des „exzessiven Computerspielens ${ }^{\text {“87 }}$. Zu deutlich geringeren Prävalenzzahlen im Sinne einer Abhängigkeit problematischer Computerspielnutzung kommt die Studie des KFN (Rehbein et al., 2009b, S. 30 40). In der Studie, die mit dem Abhängigkeitsklassifikationsinstrument KFNCSAS-I $^{88}$ arbeitete, wurden 0,8 Prozent der Schülerinnen und Schüler als abhängig, sowie weitere 1,2 Prozent der Befragten als abhängigkeitsgefährdet klassifiziert (S. 34). Damit kommen Rehbein et al., selbst bei Zusammenfassung beider als problematisch einzustufenden Spielergruppen auf - im Vergleich zu Grüsser et al. - „lediglich“ zwei Prozent Schülerinnen und Schüler mit auffälligem Computerspielverhalten. Auch in der KFN-Studie waren Jungen deutlich häufiger betroffen als Mädchen: 1,4 Prozent der männlichen Fünftklässler wurden als abhängig klassifiziert, 2,4 Prozent als abhängigkeitsgefährdet. Unter den Mädchen erschienen lediglich 0,2 Prozent als computerspielabhängig und 0,0 Prozent als abhängigkeitsgefährdet.

$\mathrm{Da}$ das in der KFN-Studie verwendete Klassifikationsinstrument in identischer Form in der KFN-Neuntklässlerbefragung 2005 von 14.301 Schülerinnen und Schülern neunter Klassen in elf deutschen Städten und Landkreisen verwendet wurde (Mößle et al., 2007, S. 113 - 126), ist hier ein Vergleich der Abhängigkeitsprävalenzdaten zwischen Fünftklässlern und Neuntklässlern möglich. In der Auswertung der Daten der KFN-Schülerbefragung 2005 wurden 3,3 Prozent der Befragten als abhängigkeitsgefährdet und 1,4 Prozent der Be-

87 Dies erscheint im Rahmen dieser Studie insofern besonders gerechtfertigt, da im Klassifikationsinstrument Kernkriterien der Computerspielabhängigkeit additiv mit Kriterien der Spielzeit verknüpft werden, wobei letztere eigentlich nicht als diagnostischer Indikator verwendet werden sollten, sondern allenfalls zur Validierung einer Klassifikation herangezogen werden sollen.

88 Im Gegensatz zum oben bereits erwähnten Nachfolger KFN-CSAS-II ist die KFN-CSASI drei Items kürzer (11 Items) und enthält lediglich Items zu den Abhängigkeitsmerkmalen „Fortsetzung trotz negativer Konsequenzen“, „Kontrollverlust“ und „Entzugserscheinungen“, während - anders als in der KFN-CSAS-II - ,,Toleranzentwicklung“ und „Einengung des Denkens und Handelns“ nicht erfasst werden (vgl. Rehbein et al., 2009b, S. 33). 
fragten als abhängig eingestuft (Baier \& Rehbein, 2009, S. 149). Insofern ergibt sich auch in diesem direkten Vergleich zweier mit demselben Instrument getesteten Stichproben unterschiedlichen Alters, dass Computerspielabhängigkeit besonders im Jugendalter eine besondere Relevanz zu haben scheint.

Rehbein et al. (2009b) konnten bereits für Schülerinnen und Schüler der fünften Klasse eine Reihe von Risikofaktoren herausarbeiten, bei deren Kumulation sich bereits im Kindesalter problematische, suchtartige Computerspielnutzungsmuster zeigen, und die spätestens im Jugendalter Prädiktoren einer Computerspielabhängigkeit sind. So zeigten die Autoren, dass die Nutzung bestimmter Spielgenres sowie bestimmte Nutzungsmotive als Indikatoren problematischen Computerspielens gelten können. Nach Ihrer Interpretation besteht ein besonderes Risiko zur Entwicklung einer späteren Computerspielabhängigkeit dann, wenn Computerspielnutzung bereits frühzeitig zur (dysfunktionalen) Kompensation realweltlicher Misserfolge eingesetzt wird und wenn bereits im Kindesalter Spiele mit klar militärischem Charakter, Spiele mit hoher Gewaltdichte sowie Onlinerollenspiele genutzt werden (S. 41 - 44). Vor diesem Hintergrund empfehlen sie die Berücksichtigung spielimmanenter Abhängigkeitsmerkmale bei der Alterseinstufung eines Computerspiels durch den gesetzlichen Jugendmedienschutz sowie eine deutliche Sensibilisierung von Computerspielherstellern, Computerspielerinnen und Computerspielern sowie Eltern minderjähriger Computerspielnutzerinnen und -nutzer, um der Entwicklung suchtartiger Spielnutzungsmuster frühzeitig zu begegnen (S. 45 - 46). 